\title{
Inference of Compact Nonlinear Dynamic Models by Epigenetic Local Search
}

\author{
William La Cava ${ }^{\mathrm{a}, *}$, Kourosh Danai ${ }^{\mathrm{a}}$, Lee Spector $^{\mathrm{b}}$ \\ ${ }^{a}$ Dept. of Mechanical and Industrial Engineering, University of Massachusetts, Amherst, USA \\ ${ }^{b}$ School of Cognitive Science, Hampshire College, Amherst, USA
}

6 Abstract

We introduce a method to enhance the inference of meaningful dynamic models from observational data by genetic programming (GP). This method incorporates an inheritable epigenetic layer that specifies active and inactive genes for a more effective local search of the model structure space. We define several GP implementations using different features of epigenetics, such as passive structure, phenotypic plasticity, and inheritable gene regulation. To test these implementations, we use hundreds of data sets generated from nonlinear ordinary differential equations (ODEs) in several fields of engineering and from randomly constructed nonlinear ODE models. The results indicate that epigenetic hill climbing consistently produces more compact dynamic equations with better fitness values, and that it identifies the exact solution of the system more often, validating the categorical improvement of GP by epigenetic local search. The results further indicate that when faced with complex dynamics, epigenetic hill climbing reduces the computational effort required to infer the correct underlying dynamics. We then apply the method to the identification of three real-world systems: a cascaded tanks system, a chemical distillation tower, and an industrial wind turbine. We analyze its solutions in comparison to theoretical and black-box approaches in terms of accuracy and intelligibility. Finally, we analyze population homology to evaluate the efficiency of the method. The results indicate that the epigenetic implementations provide protection from premature convergence by maintaining diversity in silenced portions of programs.

Keywords: system identification, genetic programming, dynamical systems, differential equations, symbolic regression

\section{INTRODUCTION}

A major goal of science is to characterize analytically the dynamic behavior of natural phenomena associated with biological, ecological, social, and economic systems, as well as the dynamics of artifacts such as wind turbines, robots, and aircraft. Dynamic behaviors are usually characterized by differential equations which in aggregate represent the dynamic model of the system. These dynamic models are the essence of the simulations that estimate/predict system behavior for policy decisions, design, optimization, control, and/or automation. This paper presents a method for construction of concise and mechanistically meaningful dynamic models from observations.

Dynamic models are preferably formulated according to first principles, to embody the knowledge of the process. However, first-principles models cannot often fully characterize the nonlinear dynamics of the process, as represented by process observations. In regress, first-principles models

*Corresponding author. Email: wlacava@umass .edu, Tel: +1 (413) 320-0544

Preprint submitted to Elsevier

June 24, 2016 
may be abandoned in favor of empirical models such as neural networks [40, 17], linear or nonlinear autoregressive moving average (ARMAX) models [36,3], or others [41, 47], that have the structural flexibility to accommodate the measured process observations. Although these empirical models provide an effective basis for estimation/prediction, they have two major drawbacks. One is their 'black-box' format which obscures the knowledge of the process acquired through adaptation. The second is their case-specificity which makes them potentially deficient in representing the process under conditions (inputs) not encompassed by the measured observations. To remedy the black-box nature of these empirical models, dynamic models consisting of differential equations can be defined in algebraic form by symbolic regression $[16,6,4]$, wherein both the structure (topology) and parameters (constants) are inferred from measured observations. Since these symbolic models are intelligible, they have the capacity to elucidate the process physics. Symbolic regression is typically conducted using genetic programming (GP) [29], which is a bio-inspired machine learning technique that constructs candidate models from mathematical building blocks and proceeds with selection, recombination and mutation over several generations before converging on a model that best fits the process observations.

In comparison to system identification methods that presume fixed model structures, symbolic regression can be computationally expensive because of its expanded search space. Furthermore, when guided solely by an error metric, it can yield unwieldy equations that are elusive to physical interpretation. To remedy these shortcomings, this paper introduces a new method of symbolic regression that fine-tunes candidate model structures by local search [32]. This fine tuning is enabled by the addition of an epigenetic layer for selection of program components (consisting of variables and instructions) to be included in the model. The incorporation of this epigenetic layer is motivated by two hypotheses: first, that the benefits of epigenetic regulation observed in biology may confer analogous improvements on GP systems; and second, that generalized local search methods enabled by epigenetics may improve the ability of GP to find correct model structures.

As to the first hypothesis, despite the highly regulated nature of biological genes, the role of epigenetics in regulating gene expressions is traditionally ignored in GP (with some exceptions, e.g. [12]). However, epigenetic processes may provide several evolutionary benefits. For example, because epigenetic processes allow the underlying genotype to encode various expressions and lead to neutral variation through crossover and mutation of non-coding segments, they may allow populations to avoid evolutionary bottlenecks or let them respond to changing evolutionary pressures [22]. Also, because they provide for phenotypic plasticity that enables gene expression to change in response to environmental pressure [10], they may allow gene expression adaptations to be inherited in offspring without explicit changes to the genotype. This property legitimizes, via epigenetic processes, once discredited ideas of Lamarck pertaining to the inheritability of lifetime adaptations $[22,20]$.

Regarding the second hypothesis, although local search methods have been developed and integrated into evolutionary algorithms [18, 63, 23, 46, 15], especially in genetic algorithms (GAs) through prescribed changes to the genotype, the role of structure optimization in symbolic regression is typically left to the GP process. Aside from some recent developments [1], local search is traditionally conducted at the genome level. More generic local search methods, like tree snipping [4], focus on improving secondary metrics like size or legibility, whereas the traditional search methods, like stochastic hill-climbing [4], linear [21] or non-linear regression [58] are confined to constant optimization. Although these local search methods improve symbolic regression performance, they cannot aid the search for program topology.

Epigenetics, on the other hand, provide a natural basis for performing local search at the 
structural (i.e., program topology) level. Motivated by this benefit of epigenetics, we introduce in this paper an epigenetics-enabled GP system to conduct topological optimization of programs at the level of gene expression. The contributions of this method are twofold: first, it introduces a generic method of topological search of the space of individual genotypes via modifications to gene expression. Second, it improves programs without affecting the genotype and without discarding the acquired knowledge gained through evolution, thereby lowering the risk of premature convergence observed in previous studies [63]. These contributions are achieved by conducting local search on the epigenome rather than the genome and making these adaptations inheritable via evolutionary processes.

The proposed Epigenetic Linear Genetic Programming (ELGP) method is tested on a large array of data generated from nonlinear ordinary differential equations (ODEs), as well as from three real-world processes, to evaluate the quality of its solutions. The paper is organized as follows. We formulate in $\S 2$ the identification problem and describe in $\S 3$ the ELGP method and its application to inference of dynamic models. We also review the relevant work in the context of GP and nonlinear dynamics modeling in $\S 4$. We then present the experimental analysis of different epigenetic implementations on a series of increasingly complex problems in $\S 5$. We begin by testing the method on a large set of data obtained from simulated nonlinear ODEs in different engineering fields, in order to illustrate its breadth of application. We then perform identification on hundreds of randomly constructed nonlinear systems, varying in complexity and dimensionality, to evaluate the scalability of the method in comparison to traditional GP approaches. Finally, we apply the ELGP method to three real-world problems, including the identification of 1) a benchmark cascaded tanks system [66], 2) a chemical distillation tower, and 3) an industrial wind turbine. The results are presented in in $\S 6$ and include comparisons of ELGP's performance in relation to other linear and nonlinear identification methods. We finish this discussion with an analysis of population diversity to study how gene expression evolves for each ELGP implementation.

\section{Problem Statement}

The underlying assumption of symbolic regression is that there exists an analytical model of the system that would generate the measured observations $y\left(t_{k}\right)$ at the sample times $t_{k}=t_{1}, \ldots, t_{N}$ under the input, $\mathbf{u}(t)$, as

$$
y\left(t_{k}, \mathbf{u}\right)=\hat{y}\left(t_{k}, M^{*}\left(\mathbf{x}, \mathbf{u}, \boldsymbol{\Theta}^{*}\right)\right)+\nu ; \quad k=1, \ldots, N
$$

where $\hat{y}$ is the model output, $\nu$ represents measurement noise in $y, \mathbf{x}=\left[x_{1}, \ldots, x_{n}\right]^{T}$ is the vector of state variables, and $M^{*}\left(\mathbf{x}, \mathbf{u}, \Theta^{*}\right)$ is the correct model form embodied by the correct parameter values $\Theta^{*}$, written $M^{*}$ hereafter for brevity. In the search for the correct model form $M^{*}$, GP typically attempts to solve the problem

$$
\text { minimize } f(M) \text { subject to } M \in \mathfrak{S}
$$

where $\mathfrak{S}$ is the space of possible models $M$, and $f$ denotes a minimized fitness function. Given that it is impractical to exhaustively search $\mathfrak{S}$, the model found to minimize $f(M)$ may only be locally optimal. For practical purposes it is assumed that a sub-optimal model can nevertheless fulfill the purpose of adequately representing the process, as depicted by the measured observations.

A common choice for estimating a candidate model output $\hat{y}(\widehat{M})$ is numerical integration or simulation of the state variables, i.e. the "output error" method [36]. However, given the sensitivity of simulation to different model structures [34] and the computational cost of numerical 
integration, the alternative approach of algebraically estimating candidate model outputs is preferred for symbolic regression $[4,49]$. In the algebraic approach, un-measured states, denoted $\tilde{\mathbf{x}}$, are estimated from measurements via numerical differentiation together with smoothing functions. In the case of first-order differential equations with un-measured state derivatives, the target is estimated numerically as $y\left(t_{k}, \mathbf{u}\right)=\tilde{\dot{x}}$, such that the prediction error of a candidate model has the form

$$
\epsilon\left(t_{k}\right)=y\left(t_{k}\right)-\hat{y}\left(t_{k}, \widehat{M}(\mathbf{x}, \mathbf{u}, \widehat{\mathbf{\Theta}})\right)=\tilde{\dot{x}}\left(t_{k}\right)-\hat{\dot{x}}\left(t_{k}\right)
$$

The fitness metric $f$ in Eq. (2) for individuals is often defined using the mean absolute error (MAE) or mean squared error (MSE), although some prefer using the correlation coefficient due to its insensitivity to linear scaling [25, 28]. We use a fitness metric [34] designed to minimize error and maximize correlation so that both the prediction error and the closeness of shapes defined by the coefficient of determination $\left(\mathrm{R}^{2}\right)$, are accounted for in the results. This fitness metric takes advantage of the covariance comparison afforded by $\mathrm{R}^{2}$ and avoids the need for post-hoc linear scaling of the solutions which could lead to increased model complexity. For target $y$ and output $\hat{y}, f$ is defined as:

$$
\begin{aligned}
f & =\frac{1}{N} \sum_{k=1}^{N}\left|\epsilon\left(t_{k}\right)\right| / R^{2}(y, \hat{y}) \\
R^{2} & =\frac{(\operatorname{cov}(y, \hat{y}))^{2}}{\operatorname{var}(y) \operatorname{var}(\hat{y})}
\end{aligned}
$$

\section{Epigenetic Linear Genetic Programming (ELGP)}

In symbolic regression, the search for candidate models is conducted by GP, wherein a population of computer programs, consisting of variables and instructions that produce models of the process, are evolved. Mathematical building blocks compose the genotype of each program that is optimized by an evolutionary algorithm. The operation steps of ELGP ${ }^{1}$, outlined in Figure 1, start with randomly constructed programs that comprise an initial population. The model outputs generated from these programs are evaluated with respect to the training data. Depending on the variant of ELGP as defined in $\S 3$, the population then undergoes some form of epigenetic adaptation. Afterwards, the population undergoes selection, recombination and mutation, as in standard GP, to produce an updated population. The process repeats until an adequate solution is produced.

The ELGP method has two salient features that improve its performance: (i) its use of linear, stack-based programs to represent equations, and (ii) its incorporation of local search of the model structures space to improve the candidate model's fitness and to reduce its complexity. These features have been shown to bolster traditional GP's performance on several benchmark regression problems in terms of the conciseness of the developed models, their fitness, and efficiency of the search $[33,32]$. The effectiveness of these features is evaluated here in construction of nonlinear dynamic models.

\subsection{GP Representation}

An innovation of the proposed ELGP method is its utilization of stack-based representation [43, $55]$ to accommodate the introduction of epigenetics. In this representation, programs are encoded

\footnotetext{
${ }^{1}$ source code available from http://www.github.com/lacava/ellen
} 


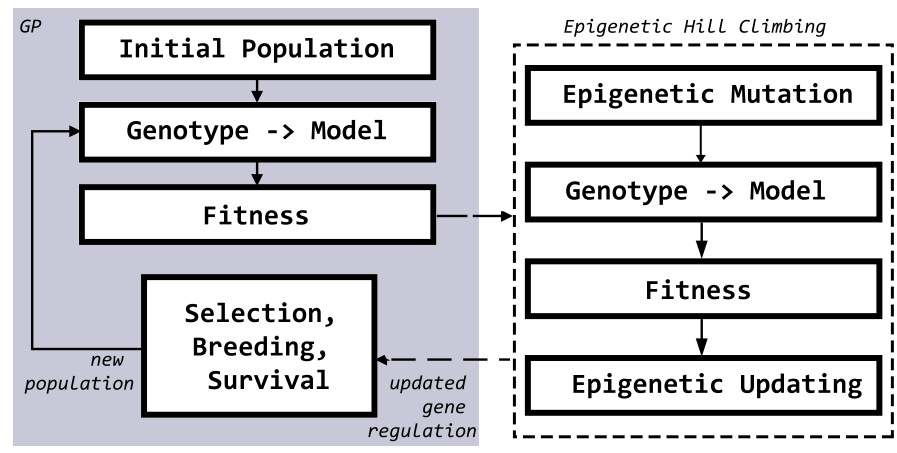

Figure 1: Block diagram of ELGP. The typical GP steps are shown on the left. After fitness evaluation and before selection, the population undergoes an iteration of epigenetic hill climbing, represented by the block on the right.

as post-fix notation, linear genotypes. This stack-based GP system is advantageous because it guarantees syntactic validity for arbitrary sequences of instructions. This property allows instructions to be silenced or activated in a genotype without invalidating the program's ability to execute, in contrast to tree-based representations that can become syntactically invalid due to changes to instructions and literals.

The syntactic robustness of the stack-based approach is achieved mainly by ignoring the execution of instructions that have an arity larger than the current size of the stack. For example, if a + operator attempts to execute and there is only one element on the stack, it does nothing. Furthermore, we base a program's behavior only on the top element of the stack after execution which allows programs to contain unused arguments. These two rules are the key to accommodating diverse program syntax. According to this flexibility, for instance, the genotypes of the following three programs $\mathbf{i}_{\mathbf{1}}, \mathbf{i}_{\mathbf{2}}$ and $\mathbf{i}_{\mathbf{3}}$ will produce the identical model $\left(x_{1}+x_{2}\right)$ :

$$
\begin{aligned}
& \mathbf{i}_{\mathbf{1}}=\left[\begin{array}{lll}
x_{1} & x_{2} & +
\end{array}\right] \Rightarrow M_{1}:\left(x_{1}+x_{2}\right) \\
& \mathbf{i}_{\mathbf{2}}=\left[\begin{array}{lllll}
x_{1} & x_{2} & + & * & *
\end{array}\right] \Rightarrow M_{2}:\left(x_{1}+x_{2}\right)
\end{aligned}
$$

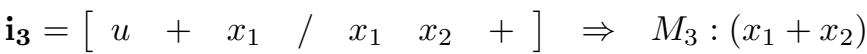

The executions of,$- *$ and / in $\mathbf{i}_{\mathbf{2}}$ are ignored due to insufficient stack size, and in $\mathbf{i}_{\mathbf{3}}$, the last element of the executed stack, $\left(x_{1}+x_{2}\right)$, is taken as the model. A step-by-step execution of program $\mathbf{i}_{3}$ is given in Figure 2 to illustrate the procedure.

\subsection{Epigenetic Learning and Evolution}

We introduce epigenetic information into the GP representation by including an on/off marker on each element in an individual's genotype. This corresponding sequence of on/off markers is referred to as an epigenome. When evaluated together, the expressed program, i.e. model, is produced by executing instructions that are on (active) and ignoring the instructions that are off (inactive). In this light, one can see that the non-coding genes (known as introns [67]) ignored in programs $\mathbf{i}_{2}$ and $\mathbf{i}_{3}$ in Eq. (6) provide local solutions to explore in the search space, making it possible to alter the topology and values of the resultant model. For example, program $\mathbf{i}_{\mathbf{3}}$ can admit several models via epigenetic transformations, including 


\begin{tabular}{|c|c|}
\hline $\mathbf{i}_{3}=\left[\begin{array}{llll}u & +x_{1} & / & x_{1}\end{array}\right.$ & $\left.x_{2}+\right]$ \\
\hline program execution & stack \\
\hline 1. $(u): \operatorname{push} u$ & {$[u]$} \\
\hline 2. (+) : ignore (insufficient argumen & {$[u]$} \\
\hline 3. $\left(x_{1}\right): \operatorname{push}\left(x_{1}\right)$ & {$\left[\begin{array}{ll}u & x_{1}\end{array}\right]$} \\
\hline 4. $(/): \operatorname{pull}\left(x_{1}\right),(u) ; \operatorname{push}\left(u / x_{1}\right)$ & {$\left[\left(u / x_{1}\right)\right]$} \\
\hline 5. $\left(x_{1}\right): \operatorname{push}\left(x_{1}\right)$ & {$\left[\left(u / x_{1}\right) \quad x_{1}\right]$} \\
\hline 6. $\left(x_{2}\right): \operatorname{push}\left(x_{2}\right)$ & {$\left[\begin{array}{lll}\left(u / x_{1}\right) & x_{1} & x_{2}\end{array}\right]$} \\
\hline 7. $(+): \operatorname{pull}\left(x_{2}\right),\left(x_{1}\right) ; \operatorname{push}\left(x_{1}+x_{2}\right)$ & {$\left[\begin{array}{ll}\left(u / x_{1}\right) & \left(x_{1}+x_{2}\right)\end{array}\right]$} \\
\hline$\rightarrow$ return last element on stack & $M_{3}:\left(x_{1}+x_{2}\right)$ \\
\hline
\end{tabular}

Figure 2: Stack-based execution of GP program $\mathbf{i}_{3}$ from Eq. (6). Arguments are pushed to the stack and operands $(*,+$, etc.) pull arguments from the stack, perform an operation, and push the result. Operands without sufficient arguments are ignored, and the final element on the stack at the end of execution is returned as the model.

$$
\begin{aligned}
\mathbf{i}_{3} \rightarrow \mathbf{i}_{3}^{\prime} & =\left[\begin{array}{llllccc}
1 & 1 & 0 & 0 & 0 & 1 & 1 \\
u & + & x_{1} & / & x_{1} & x_{2} & +
\end{array}\right] \Rightarrow M_{3}^{\prime}:\left(u+x_{2}\right) \\
\mathbf{i}_{3} \rightarrow \mathbf{i}_{3}^{\prime \prime} & =\left[\begin{array}{lllllll}
1 & 1 & 1 & 1 & 0 & 0 & 0 \\
u & + & x_{1} & / & x_{1} & x_{2} & +
\end{array}\right] \Rightarrow M_{3}^{\prime \prime}:\left(u / x_{1}\right) \\
\mathbf{i}_{3} \rightarrow \mathbf{i}_{3}^{\prime \prime \prime} & =\left[\begin{array}{llllccc}
1 & 0 & 1 & 1 & 0 & 1 & 1 \\
u & + & x_{1} & / & x_{1} & x_{2} & +
\end{array}\right] \Rightarrow M_{3}^{\prime \prime \prime}:\left(u / x_{1}+x_{2}\right)
\end{aligned}
$$

Similarly, program $\mathbf{i}_{2}$ in Eq. (6) admits the models $\left(x_{1}+x_{2}\right),\left(x_{1}-x_{2}\right),\left(x_{1} * x_{2}\right)$, and $\left(x_{1} / x_{2}\right)$ via epigenetic transformations.

During the ELGP process depicted in Figure 1, the epigenetic markers are initialized randomly (in the initial population) with a probability of being active. We use $50 \%$ as the initial probability for the experimental studies in $\S 5$, chosen according to a previously conducted parametric study [33]. The extent to which epigenetic information is learned and inherited is a research question that we address by exploring different implementations. The simplest topological search method within ELGP is Ep1M, which mutates the epigenetic layer of each individual each generation; the hill climber in Figure 1 is skipped. Thus for Ep1M, epigenetic mutations face only evolutionary pressures. In contrast, the epigenetic hill climbing (EHC) cases EHC1, EHC5 and EHC10 use the epigenetic information explicitly to improve individuals each generation (the EHC is described in §3.2.2). The three methods execute one, five and ten iterations of EHC each generation, respectively. Two control methods, Base and Ep0, are used for comparison. In the Base case, individuals are represented as basic genotypes as in Eq. (6). The Ep0 case acts like Base but with half of the genes in the initial code permanently silenced. As such Ep0 accounts for the effect that smaller active programs and explicit introns might have. Neither Base nor Ep0 use the right half of the system in Figure 1 (i.e. the program never enters epigenetic mutation).

\subsubsection{Epigenetic Mutation}

Whitley et al. [63] introduced Lamarckian updating to GAs by conducting local search of the bit strings within 1 Hamming distance of the current bit string. In theory it would be possible to treat the epigenome as a bit string and proceed similarly. However, the cost of GP fitness evaluations 


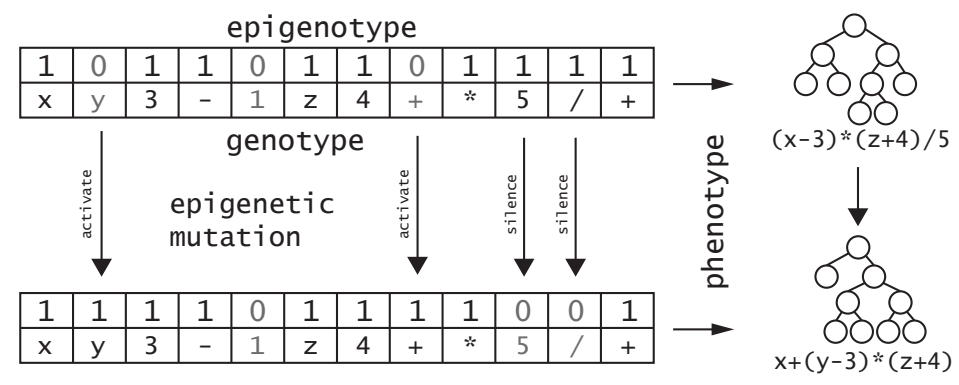

Figure 3: Illustration of an epigenetic mutation applied to a GP program. The mutations result in topological changes to the model (phenotype), shown on the right.

may render this approach intractable. Instead, each generation, the epigenome is uniformly mutated with a probability of $10 \%$ at each gene. The mutation flips the binary value of the epigenome at the gene, thus activating or silencing that gene. The operation is uniform with respect to the number of instructions. Epigenetic mutation is illustrated in Figure 3 to show how these epigenetic changes can result in significant topological changes to the resultant models. For the EHC1, EHC5 and EHC10 implementations, the epigenetic mutation is followed by hill climbing, described next.

\subsubsection{Epigenetic Hill Climbing}

In order to mimic the acquisition of lifetime learning by epigenetic adaptation, the EHC implementations evaluate epigenetic changes $\mathbf{i} \rightarrow \mathbf{i}^{\prime}$ to determine whether individuals should be updated. At each iteration of epigenetic mutation, EHC1, EHC5 and EHC10 test the changes to the model for acceptance. Epigenetic changes to an individual are kept only if the fitness is improved or not changed, i.e. $f_{\mathbf{i}^{\prime}} \leq f_{\mathbf{i}}$ (fitness $f$ is being minimized).

In addition, we break fitness ties by preferring less complex equations. Model complexity can be represented by several approaches. For example, one can count the number of nodes in the parse tree, calculate the order of a Chebyshev polynomial fit to the model's output [62], or recursively aggregate the complexity of sub-expressions [27]. Here, we account for model complexity by assigning component function nonlinearities to genotype components [53]. The complexity $C_{\mathbf{i}}$ of program $\mathbf{i}$ with active genotype $\mathbf{g}_{\mathbf{a}}=\left[\begin{array}{lll}g_{a_{1}} & \ldots & g_{a_{\ell}}\end{array}\right]$ is defined as $C_{\mathbf{i}}=\sum_{q=1}^{\ell} c\left(g_{a_{q}}\right)$, where component function nonlinearities [53] are defined as

$$
c\left(g_{a}\right)=\left\{\begin{array}{rrr}
4 & : & \left(g_{a}=\log \right) \vee\left(g_{a}=\exp \right) \\
3 & : & \left(g_{a}=\sin \right) \vee\left(g_{a}=\cos \right) \\
2 & : & \left(g_{a}=/\right) \vee\left(g_{a}=\sqrt{()}\right) \\
1 & : & \text { otherwise }
\end{array}\right.
$$

Lower-complexity programs with equivalent fitness are accepted, giving the condition

$$
\text { pass }=\left(f_{\mathbf{i}^{\prime}}<f_{\mathbf{i}}\right) \vee\left(\left(f_{\mathbf{i}^{\prime}}=f_{\mathbf{i}}\right) \wedge\left(C_{\mathbf{i}^{\prime}}<C_{\mathbf{i}}\right)\right)
$$

If the epigenetically mutated individual $\mathbf{i}^{\prime}$ does not pass Eq. (9), the changes are discarded and $\mathbf{i}$ is kept in the population. Otherwise $\mathbf{i}$ is replaced with $\mathbf{i}^{\prime}$. 


\subsubsection{Epigenetic Inheritance}

A key feature of ELGP is the inheritance of epigenetic values throughout the evolutionary process. During crossover, the epigenetic values of the parent genes are kept intact such that the child receives the epigenetic states of the genes it has inherited. If a new gene is introduced via genetic mutation, that gene has the same probability of being active as the initial genes of the population $(50 \%$, in the current study).

\section{Related Work}

There has been some work to incorporate epigenetic learning into GP, notably by Tanev [57]. In that case the focus was to model histone modification through a double cell representation as demonstrated in a predator-prey problem. Unlike our approach, Tanev did not treat lifetime epigenetic modifications as inheritable, as is supported by recent studies in biology [61, 24, 11]. There have also been a number of studies on the effects of non-coding segments in GP, some of which have found that the structural presence of introns protect genotypes from destructive crossover operations (i.e., operations that produce children less fit than their parents) $[42,67,5]$. Non-coding segments were found to be useful in evo-devo for evolution of arbitrary shapes as well [14]. In each of these studies, introns were declared explicitly or measured during evolution, rather than being actively manipulated by the system itself as in ELGP. Our preliminary study of epigenetic initialization finds rates of beneficial crossover to be the highest with the probability set to $50 \%$ [33].

In addition, several GP systems use similar stack-based or linear genome representations, such as PushGP [55], Push-forth [26] and Gene Expression Programming [12], that could trivially implement the epigenetic layer incorporated in the ELGP method. Similarly, there are methods that leverage neutrality (i.e., different genotypes with the same fitness) by creating a genotype - phenotype mapping; e.g., Cartesian GP [39] and Binary GP [2]. Developmental approaches to GP [30] also make use of genotype - phenotype mappings. Our goal with ELGP is to incorporate local search of gene expression as a viable, generic GP extension that does not require large changes to implement. As mentioned earlier, there are a plethora of studies on local search methods for improving GP by Lamarckian or Baldwinian means, yet very few have considered these changes to occur at the epigenetic level instead of the genotype level. A notable exception is Multiple Regression GP [1], in which parameter values are implied at each node location and updated by linear regression. Still, the tangible improvements brought about by this and most other local search methods for symbolic regression are achieved by parametric, rather than topological, search.

Several methods based on GP have been proposed for modeling nonlinear dynamic systems, including ODE model structures [16, 6, 4, 49] and GP-NARMAX models [44, 45]. GP populations are often evolved with multi-objective methods like SPEA2 [68] and NSGA-II [9] to pressure model size. Unlike ELGP, these techniques for compact modeling focus on changes to the core GP algorithm (selection and fitness evaluation). In this regard, ELGP could be readily applied in those proposed frameworks. The multiobjective framework we use in our experiments is that proposed in [50], as discussed in $§ 5.1$.

More broadly, GP is one approach to nonlinear system identification, among many others. A common approach embodied by Hammerstein-Weiner and nonlinear auto-regressive modeling with exogenous inputs (NARX) is to combine a chosen nonlinear transformation (or transformations) with a linear model. Although the use of a nonlinear estimator can increase the capacity compared to ARX modeling, per say, in both cases the structure of the nonlinearity must be specified beforehand, 
contrary to symbolic regression. These approaches also produce complex models that can obfuscate intuitive explanations of their predictive power. To remedy this, greedy structure selection methods have been proposed for nonlinear polynomial models [19], notably for the NARMAX model using the orthogonal least squares (OLS) [7,3]. GP methods have also been proposed to optimize the structural identification of OLS models [38]. The goal of ELGP is to improve the ability of GP representations to produce intelligible model structures, which in turn can be applied to autoregressive representations [31] and/or coupled with a desired parameter estimation strategy $[21,58$, $28]$.

\section{Experimental Methods}

We describe in this section the evolutionary framework to which ELGP is applied and the settings that are used to conduct the experiments, followed by a description of the set of problems that are used to compare the performance of each GP treatment. $\S 5.1$ describes the algorithms used to perform selection and search operations within GP, which build upon previous symbolic regression research. In $\S 5.2$, we describe implementation optimizations related to efficiently performing hill climbing on epigenetically mutated programs. Finally in $\S 5.3$ we present the set of problems on which ELGP is evaluated, which include simulated ODEs from various fields, randomly constructed ODEs that vary in complexity, and lastly a real-world nonlinear dynamics modeling application based on data from a benchmark cascaded tanks system.

\subsection{Evolutionary Algorithm}

Several state-of-the-art symbolic regression tools leverage Pareto optimization for selection and survival [53, 49], and our preliminary tests (not reported here) confirm that the age-fitness Pareto survival method [50] outperforms traditional GP [29] on several problems. In an effort to demonstrate ELGP on a high-performance configuration, we use age-fitness Pareto survival as the evolutionary algorithm in our experiments. In this scheme, each individual is assigned an age equal to the number of generations since its oldest ancestor was created. Each generation, a new individual is introduced to the population as a means of random restart. Selection for breeding is random, and during breeding a number of children equal to the overall population size is created. At the end of each generation, environmental selection is conducted according to the Strength Pareto Evolutionary Algorithm 2 (SPEA2) [68] to reduce the size of the set $P$ comprising the current population plus the newly created individuals down to the original population size $N$. Note that the hypervolume method from NSGA-II [9] could also be used for this task, although previous work suggests that SPEA2 performs better in low dimensions [68].

SPEA2 uses two measures to perform this reduction of $P: 1$ ) Pareto strength of an individual, $S(\mathbf{i})$, which is the number of individuals equal to or dominated by $\mathbf{i}$, divided by $|P|+1$, and 2 ) a density estimate $D(\mathbf{i})<1$, based on the inverse of the distance to the $k$-th nearest neighbor [52] of $\mathbf{i}$ in objective space (in this case the objectives are normalized between zero and one). These metrics are used to define a fitness value $F(\mathbf{i})$ that combines the total strength of the individuals $\mathbf{j} \in P$ that dominate $\mathbf{i}$, denoted as $\mathbf{j} \prec \mathbf{i}$, with density estimate $D(\mathbf{i})$, as

$$
F(\mathbf{i})=\sum_{j \in P, j \prec i} S(\mathbf{j})+D(\mathbf{i})
$$

Every nondominated solution is first copied to the new population. If the new population size is smaller than $N$, individuals are added in order of lowest $F(\mathbf{i})$. If the population is larger than $N$, 
signifying that there are more than $N$ nondominated solutions, individuals are removed iteratively based on $D(\mathbf{i})$. In the latter scenario, the use of $D(\mathbf{i})$ for selection helps preserve spread of solutions along the Pareto front.

A uniform alternation crossover operator is used to produce two children from two parents, as in [54]. The mutation operator is applied uniformly to the chosen parent with a probability of $2.5 \%$ at each gene. If a constant gene is picked for mutation and ephemeral random constants (ERCs) are being used, the constant is perturbed by Gaussian noise with standard deviation equal to half the magnitude of the constant. Otherwise the instruction is mutated to a randomly chosen gene.

In order to optimize the parameters (constants) of the models, one iteration of stochastic hill climbing is conducted on model parameters each generation. The hill climber perturbs all constant values in the active genotype by Gaussian noise with a standard deviation equal to $10 \%$ of the value of the constant. These changes are kept if they result in a better fitness for the individual. This method of constant optimization is chosen due to its lightweight nature compared to least-squares approaches.

Each trial was allocated a maximum number of point evaluations, i.e., gene executions, to normalize for the different program sizes among methods. A GP run will exit early if the fitness condition $f<10^{-6}$ is achieved before the designated number of point evaluations has been reached. We observed this fitness termination condition to be sufficient for reaching exact solutions for the problems studied here.

\subsection{Optimizations}

The following optimization provisions are applied to ELGP in order to reduce the number of point evaluations required to evaluate the fitness of an individual that has undergone epigenetic mutation. The majority of run-time in most GP systems (including ours) is devoted to fitness evaluation. This motivates reduction of the number of point evaluations required.

Fitness Escape. EHC requires additional fitness evaluations in order to determine whether the prescribed epigenetic changes will be kept. Given that the fitness $f_{\mathbf{i}}$ of program $\mathbf{i}$ cannot decrease with the evaluation of more fitness cases $k_{1 \ldots n}$, evaluation of the epigenetically mutated individual $\mathbf{i}^{\prime}$ can be halted if at any point $f_{\mathbf{i}^{\prime}}\left(1 \ldots k_{j}\right)>f_{\mathbf{i}}\left(1 \ldots k_{n}\right)$ for $1 \leq j<n$. This allows $\mathbf{i}^{\prime}$ to be discarded before its fitness is fully evaluated because it is guaranteed to be worse than $\mathbf{i}$. Since fitness is always equal to or larger than MAE (see Eq. (4)), the halt condition can be defined conservatively using the mean absolute error (MAE) of $\mathbf{i}^{\prime}$ and the fitness of $\mathbf{i}$ as

$$
\text { halt }=\frac{1}{N} \sum_{k=1}^{j}\left|y\left(t_{k}\right)-y\left(t_{k}, M_{\mathbf{i}^{\prime}}\right)\right|>f_{\mathbf{i}}
$$

Stack Tracing. In GP tree representations, the output of a node in the program typically depends only on the outputs of its child nodes (and those children's children and so forth). We can say conservatively with ELGP representations that no instruction in the stack is dependent on an instruction to its right. Therefore, when a gene is silenced or activated, only the outputs of the genes to its right in the genotype are affected, hence only part of the program needs to be reevaluated. To avoid repeated instruction evaluations during epigenetic hill climbing, we save the intermediate program outputs of each gene, and after epigenetic mutation reevaluate only those genes to the right of the left-most location of mutation. 
Table 1: ELGP system settings as applied to the Textbook ODE problems.

\begin{tabular}{lr}
\hline General Settings & Value \\
\hline Population size & 1000 \\
Crossover / Mutation & $80 / 20 \%$ \\
Program length limits & {$[3,50]$} \\
ERC range & {$[-10,10]$} \\
Termination criterion & $2.5 \mathrm{E} 10$ point evals or $f<1.0 \mathrm{E}-6$ \\
Trials & 100 \\
Function set & $\{+,-, *, /, \sin , \cos \}$ \\
\hline
\end{tabular}

Saving the stack outputs is a trade-off between memory and time resources since it requires more memory to save the intermediate outputs but requires fewer point evaluations to evaluate epigenetically mutated individuals. The trade-off is favorable in our implementation because processor resources are much more limited than memory resources. Similar partial evaluation strategies have been proposed, e.g., in [35].

\subsection{Problems}

The methods are first compared on a set of coupled, two-state nonlinear ODEs adapted from [56] and proposed in [51]. Second, they are tested for scalability against a suite of hundreds of randomly generated ODE problems with varying complexity and dimensionality. Finally, ELGP is applied to the identification of nonlinear dynamics of three real-world processes, including a pump-fed system of cascaded water tanks, an industrial chemical distillation tower, and an industrial wind turbine.

\subsubsection{Textbook ODE problems}

The textbook ODE problems represent seven two-state, nonlinear systems from the fields of biology, electrical engineering, physics, ecology, and fluid dynamics. For brevity, the form of the models is shown alongside identification results in Table 3. In accordance with [51], each system is simulated for 10 seconds from 4 different initial conditions chosen randomly within stable basins of attraction, giving a total of 400 data points for training. The settings for each problem are summarized in Table 1. In order to give a measure of nonlinearity and/or difficulty of these identification problems, we also use multiple linear regression (LR) to estimate models for these systems. The LR models are estimated as a weighted sum of the states (in this case the systems have no external inputs), i.e. $\hat{y}=\hat{\beta}^{T} \mathbf{x}$, where $\hat{\beta}$ is the least-squares solution minimizing the sum of the squared prediction error: $\sum_{k=1}^{N}\left(y\left(t_{k}\right)-\hat{\beta}^{T} \mathbf{x}\left(t_{k}\right)\right)^{2}$.

\subsubsection{ODE suite}

In order to test the scalability of the methods, random ODE systems were generated of varying size (nodes) and dimensionality (number of variables). This approach to scalability testing is used in order to remove problem selection bias and to quantify the methods' performance with different target complexity $[48,8]$. The dynamic systems were generated in the following fashion. First, differential equations were randomly generated using the same equation generation technique that initializes populations of equations. Second, the equations were simulated as first-order differential equations (using Runge-Kutta 4) according to a random set of initial conditions from the range $[-5,5]$. The outputs of these simulation runs were included in the training set. The validation set was subsequently generated by simulating the equations with initial conditions randomly selected from the range $[-10,10]$. Equations that produced invalid outputs were discarded. Finally, the 
Table 2: ODE suite problem settings.

\begin{tabular}{lr}
\hline \multicolumn{2}{c}{ ODE Suite Settings } \\
\hline Number of Nodes & 3 to 33 \\
Dimensions & 1 to 8 \\
Models per setting & 5 \\
Trials per model & 10 \\
Total models & 640 \\
Total trials & 6400 \\
Function set & $\{+,-, *, /, \sin , \cos , \exp , \log \}$ \\
\hline
\end{tabular}

valid equations were simplified symbolically in MATLAB in order to determine their most succinct representation, and binned by number of nodes and dimensions. The result of the entire process was 640 unique ODE problems of 3 to 33 nodes and 1 to 8 variables that were subjected to 10 trials of identification, for a total of 6,400 trials per GP treatment. The ODE suite settings are summarized in Table 2 .

\subsubsection{Real-world Applications}

We consider three real-world applications of ELGP. The first is a benchmark cascaded tanks system consisting of three measured variables. The second two come from complex industrial processes with 25 (chemical tower) and 8 (wind turbine) measured variables. All three processes are known to exhibit nonlinear dynamics.

In order to analyze ELGP's performance in the context of other nonlinear modeling approaches, we compare the quality of the ELGP's solutions to these problems with solutions from standard linear and nonlinear empirical modeling approaches. The models were developed using ARX and two NARX approaches with different nonlinear transformations: wavelet networks (NARX-W) and feedforward neural networks (NARX-NN). We used the MATLAB System Identification toolbox [37] to generate these models using default settings. For the wavelet network, the set of nonlinear regressors was computed using a radial wavelet expansion with an automatically determined number of terms. For the NARX-NN, a feedforward network with 10 hidden neurons was constructed and trained with back-propagation learning using the Levenberg-Marquardt algorithm. In order to provide uniformity among the predicted outputs of these models and that of ELGP's solutions, the ELGP models were simulated on the test sets, such that prediction error (Eq. (3)) was defined in terms of the simulated output $\hat{y}\left(t_{k}\right)$ rather than the state derivatives.

Benchmark System. In order to study the performance of ELGP on a real-world benchmark problem, we performed identification based on a set of observations collected from two cascaded tanks fed by a water pump [66]. Using the Bernoulli principle and mass conservation, this system can be represented by the following nonlinear equations:

$$
\begin{aligned}
& \dot{h}_{1}=-\theta_{1} \sqrt{h_{1}}+\theta_{2} u(t)+w_{1}(t) \\
& \dot{h}_{2}=\theta_{1} \sqrt{h_{1}}-\theta_{3} \sqrt{h_{2}}+w_{2}(t) \\
& y_{1}=h_{1}(t)+e_{1}(t) \\
& y_{2}=h_{2}(t)+e_{2}(t)
\end{aligned}
$$

where $\theta_{1}=-\frac{a_{1} \sqrt{2 g}}{A_{1}}$, and $\theta_{2}=-\frac{k}{A_{1}}, \theta_{3}=\frac{a_{2} \sqrt{2 g}}{A_{2}}$. States $h_{1}$ and $h_{2}$ represent the water levels in the upper and lower tanks, respectively; $a_{1}$ and $a_{2}$ are the outlet areas; $A_{1}$ and $A_{2}$ are the horizontal 
cross sections of the tanks; $g$ is the gravitational constant; $k$ is the pump voltage to flow conversion constant; $w_{1}(t)$ and $w_{2}(t)$ are system noise; and $e_{1}(t)$ and $e_{2}(t)$ are measurement noise.

The data set comprises 2500 samples, acquired at a sampling period of 5 seconds. We divided this set 50/50 for training and testing. These data have been proposed for benchmarking nonlinear system identification approaches [64] and are freely available [65]. For ELGP, we use EHC5 with the settings of Table 1 and an increased function set $\{+,-, *, /, \sin , \cos , \exp , \log , \sqrt{()}\}$. For ARX and NARX, identification of these models was performed as a first-order function of the input, i.e. with the regressors $y_{1}\left(t_{k}-1\right), y_{2}\left(t_{k}-1\right)$, and $u\left(t_{k}\right)$.

Industrial Processes. In addition to finding exact solutions to known systems, ELGP should be able to identify reliable models of real-world systems for which no true model is known. To this end, two complex industrial problems are also considered in this work. The first is the Tower problem ${ }^{2}$, consisting of a set of 15-minute averaged time series data taken from a chemical distillation tower, totaling 3135 samples. The goal is to predict the propelyne concentration at the top of the tower from 25 measured variables. The second problem, referred to as the Wind problem, features data collected from the Controls and Advanced Research Turbine, a $600 \mathrm{~kW}$ wind turbine operated by the National Wind Technology Center [13]. The data set consists of 6000 time-series measurements of wind speed, control actions, and acceleration measurements that are used to predict the bending moment measured at the base of the wind turbine.

For these two problems, solutions are formulated as fifth-order discrete-time dynamic models of the form $\hat{M}\left(\hat{y}\left(t_{k}-1\right) \ldots \hat{y}\left(t_{k}-5\right), \zeta\left(t_{k}\right) \ldots \zeta\left(t_{k}-5\right), \hat{\mathbf{\Theta}}\right)$ in terms of model outputs $\hat{y}$, measured variables $\zeta$, and model parameters $\hat{\boldsymbol{\Theta}}$. This formulation was used with the ELGP, ARX and NARXNN methods.

\section{Results and Discussion}

We first present results obtained on the textbook ODE problems in $\S 6.1$. Comparisons include the number of exact solutions, the fitness of the best solutions, and complexity of the best models found by each method. Next we analyze in $\S 6.2$ the ODE suite results according to fitness as a function of point evaluations in training and testing over the entire suite. To give a sense of the scalability of the methods, we group the results by target complexity and compare the number of solutions found and the computational effort spent to reach these solutions. We then compare the ELGP solution with a few black-box models obtained from the experimental data of a real world benchmark identification problem in $\S 6.3$, and compare the ELGP's solution with the theoretical model of the system. We end our analysis with a detailed look at population diversity of the Bacterial Respiration problem, which provides insight into the mechanics of the variants of ELGP (Ep1M, EHC1, EHC5, EHC10), particularly the EHC methods (EHC1, EHC5, EHC10), that lead to the better solutions obtained on many of the studied problems.

\subsection{Textbook ODE problems}

As a preliminary evaluation of the intelligibility of ELGP's solutions, the most frequent solutions to the textbook ODE problems that were found using Base and the ELGP variant EHC5 are compared against the target model forms in Table 3. Particularly noteworthy are the differences

${ }^{2}$ http://symbolicregression.com/?q=towerProblem 
Table 3: The textbook ODE problems (left). The models generated by Base and ELGP variant EHC5 are shown on the right.

\begin{tabular}{|c|c|c|c|}
\hline System & Target & Base Most Frequent Solution & EHC5 Most Frequent Solution \\
\hline \multirow[t]{2}{*}{$\begin{array}{l}\text { Bacterial } \\
\text { Respiration }\end{array}$} & $\dot{x}=20-x-\frac{x \cdot y}{1+0.5 \cdot x^{2}}$ & $\begin{array}{l}\dot{x}=-(7.006 \cdot(x+0.4722 \cdot y- \\
31.11)) / y\end{array}$ & $\dot{x}=20.12-1.009 \cdot x-\frac{1.979 \cdot y}{x}$ \\
\hline & $\dot{y}=10-\frac{x \cdot y}{1+0.5 \cdot x^{2}}$ & $\begin{array}{l}\dot{y}= \\
9.994-0.5669 \cdot y \cdot \sin (3.526 / x)\end{array}$ & $\dot{y}=10.0-\frac{2.0 \cdot x \cdot y}{2.001+x^{2}}$ \\
\hline \multirow[t]{2}{*}{ Bar Magnets } & $\dot{\theta}=0.5 \cdot \sin (\theta-\phi)-\sin (\theta)$ & $\begin{array}{l}\dot{\theta}=\sin (\sin (\cos (\cos (\theta)-\phi)+ \\
\sin (\theta)-3.65))\end{array}$ & $\dot{\theta}=0.5 \cdot \sin (\theta-\phi)-\sin (\theta)$ \\
\hline & $\dot{\phi}=0.5 \cdot \sin (\phi-\theta)-\sin (\phi)$ & $\dot{\phi}=-0.5 \cdot \sin (\theta-\phi)-\sin (\phi)$ & $\dot{\phi}=-0.5 \cdot \sin (\theta-\phi)-\sin (\phi)$ \\
\hline \multirow[t]{2}{*}{ Glider } & $\dot{v}=-0.05 \cdot v^{2}-\sin (\theta)$ & $\dot{v}=-0.05 \cdot v^{2}-\sin (\theta)$ & $\dot{v}=-0.05 \cdot v^{2}-\sin (\theta)$ \\
\hline & $\dot{\theta}=v-\cos (\theta) / v$ & $\dot{\theta}=v-(\cos (\theta)) / v$ & $\dot{\theta}=v-(\cos (\theta)) / v$ \\
\hline \multirow[t]{2}{*}{$\begin{array}{l}\text { Lotka-Volterra } \\
\text { interspecies } \\
\text { dynamics }\end{array}$} & $\dot{x}=3 \cdot x-2 \cdot x \cdot y-x^{2}$ & $\dot{x}=3.0 \cdot x-2.0 \cdot x \cdot y-x^{2}$ & $\dot{x}=3.0 \cdot x-2.0 \cdot x \cdot y-x^{2}$ \\
\hline & $\dot{y}=2 \cdot y-x \cdot y-y^{2}$ & $\dot{y}=2.0 \cdot y-x \cdot y-y^{2}$ & $\dot{y}=2.0 \cdot y-x \cdot y-y^{2}$ \\
\hline \multirow[t]{2}{*}{ Predator Prey } & $\dot{x}=x \cdot\left(4-x-\frac{y}{1+x}\right)$ & $\begin{array}{l}\dot{y}=-0.5674 \cdot x \cdot(y+x \\
\left.\cos \left(\frac{0.7896 \cdot y}{x+\cos (0.1632 \cdot x)}\right)-6.119\right)\end{array}$ & $\begin{array}{l}\dot{y}=0.3516 \cdot x \cdot \\
\left(8.122-\frac{x}{\cos (x / y)}-y\right)\end{array}$ \\
\hline & $\dot{y}=y \cdot\left(\frac{x}{1+x}-0.075 \cdot y\right)$ & $\begin{array}{l}\dot{y}=(y+0.01059) \\
\left(\frac{x}{x+1.004}-0.07488 \cdot y\right)\end{array}$ & $\dot{y}=y \cdot\left(\frac{x}{1.0+x}-0.075 \cdot y\right)$ \\
\hline \multirow[t]{2}{*}{ Shear Flow } & $\dot{\theta}=\cot (\phi) \cdot \cos (\theta)$ & $\dot{\theta}=\cot (\phi) \cdot \cos (\theta)$ & $\dot{\theta}=\cot (\phi) \cdot \cos (\theta)$ \\
\hline & $\begin{array}{l}\dot{\phi}=\left(\cos ^{2}(\phi)+0.1 \cdot \sin ^{2}(\phi)\right) \\
\sin (\theta)\end{array}$ & $\begin{array}{l}\dot{\phi}= \\
0.45 \cdot \sin (\theta) \cdot(\cos (2.0 \cdot \phi)+1.222)\end{array}$ & $\begin{array}{l}\dot{\phi}=2.076 \\
\sin (\cos (\sin (\phi))-0.4918) \\
\sin (\theta)\end{array}$ \\
\hline \multirow[t]{2}{*}{$\begin{array}{l}\text { van der Pol } \\
\text { oscillator }\end{array}$} & $\dot{x}=10 \cdot\left(y-\frac{1}{3} \cdot\left(x^{3}-x\right)\right)$ & $\begin{array}{l}\dot{x}=(y+10.41) \cdot(2.232 \\
\sin (x)-1.879 \cdot x+\sin (y))\end{array}$ & $\dot{x}=10.0 \cdot\left(y-0.333 \cdot\left(x^{3}-x\right)\right)$ \\
\hline & $\dot{y}=-\frac{1}{10} \cdot x$ & $\dot{y}=-0.1 \cdot x$ & $\dot{y}=-0.1 \cdot x$ \\
\hline
\end{tabular}

observed between Base the and EHC5 solutions for the Bacterial Respiration (1 and 2), Bar Magnets (1), Predator Prey (1 and 2), and van der Pol oscillator (1) identifications. In each of these cases, EHC5 more often identifies the exact solution, or at least an approximation of it that is less complex than that inferred by Base. In some cases, e.g. Bacterial Respiration 1 and van der Pol 1, the approximate solutions from EHC5 have most of the terms of the target correct, and thus form a sensible approximation of the true system. Note that the Base solution to Predator Prey 2, i.e.,

$$
\dot{y}=(y+0.01059) \cdot\left(\frac{x}{x+1.004}-0.07488 \cdot y\right)
$$

could be made more correct through the change $(y+0.01059) \rightarrow y$. This type of topological model change is easily reached via epigenetic transformations, and, as is shown in Table 3, EHC5 more frequently identifies the underlying target model for this problem.

A central goal of ELGP is to bolster the performance of GP in system identification through local topological search. To this end, the number of solutions, median best fitness and average equation size (number of active nodes) for the different methods are summarized in Tables 4 and 5. Pairwise statistical comparisons are given for each result. Note that three of the identification tasks (Glider 2, Shear Flow 1, and van der Pol 2) are exactly identified $100 \%$ of the time by every GP treatment, suggesting that they are easy for GP to solve. For the eleven other problems, the results show that the training and test fitnesses and solution counts are improved by EHC. For 
example, on each of these eleven problems, the ELGP variant EHC10 finds significantly $(p<0.05)$ more exact solutions and produces models with better training and test fitnesses than Base, Ep0 or Ep1M, as indicated by highlighting, bold text, and * in the tables. In terms of fitness, EHC5 provides a significant improvement relative to Base on eight out of eleven and EHC1 performs better on five out of eleven problems, thus suggesting that results improve with more iterations of EHC. Among the eleven more difficult problems, all of the EHC methods perform significantly better than Ep0 or Ep1M, in terms of fitness as well as exact solutions. Overall the results of Ep0 and Ep1M show a marginal to negative difference in estimation capacity compared to Base. The guided search provided by EHC, therefore, is key to the observed improvements.

The results indicate that every GP method produces better models than linear regression (LR) for these problems (with the exception of the linear second state of the van der Pol problem), which is to be expected given the known nonlinear nature of this set of problems. However, LR has the advantage of quick training times, with median convergence times on the order of 0.01 second for these problems, as shown in the last column of Tables 4 and 5. The GP methods converge to the minimum fitness model in approximately 1 to 100 seconds, depending on the difficulty of the problem. It is worth noting that the EHC methods do not increase the computation time compared to Base, and occasionally decrease it, which can be attributed to the lower proportion of optimization spent conducting GP generations and the higher proportion spent in EHC. The majority of ELGP computation times are not significantly different from Base. These convergence times suggest that the proposed identification methods may be suitable for certain online applications, depending on the time window constraints between model updates.

In addition to improving predictive ability, a motivation for the ELGP design is the delivery of concise solutions. This property is evident from the average program sizes in Tables 4 and 5 . To further evaluate this aspect of the results, in Figure 4, the best solutions of the 100 trials are evaluated in terms of Solution Bloat, defined as the difference in complexity (Eq. (8)) between the GP solution and the target. These results show that the ELGP variants all produce solutions that are more succinct than those achieved by Base. Among ELGP variants, Ep0 and Ep1M produce the most succinct models. The EHC methods also produce more succinct models with less solution bloat than Base; however, it is observed that the hill climbing aspect of EHC leads to slightly larger models than Ep0 or Ep1M. Nevertheless, in addition to producing more succinct models than Base, the EHC methods find exact solutions more often for most problems, as shown in Tables 4 and 5 .

\subsection{ODE suite}

To evaluate the ability of ELGP to scale to problems of increasing complexity, we evaluate the performance of the treatments Base, Ep0, Ep1M, EHC1 and EHC5 on a suite of 640 randomly generated target systems. The best fitness on training and test sets for the entire ODE suite is shown in Figures 5 and 6 as a function of point evaluations. The results indicate the better fitness minimization properties of the ELGP variants EHC1 and EHC5. The number of solutions found, and the computational effort to reach those solutions, are plotted in Figures 7 and 8, respectively. The results are broken into groups based on the number of arguments and operands (i.e. nodes) in the solution equation, for example 7, 9, 11, and so on. Figure 7 demonstrates the ability of the ELGP variants, especially EHC5, to find more solutions than Base or Ep0, and the difference in performance grows as the number of nodes in the solution increases, although the overall number of solutions decreases with more complex targets. In addition, EHC1 and EHC5 tend to find solutions with less computational effort than the other methods, as demonstrated in Figure 8, where we plot the number of point evaluations to termination for the trials in which exact solutions were found. 
Table 4: Comparison of best-of-run results for the ODE benchmark problems. Statistical significance $(p<0.05)$ is denoted as follows: ${ }^{\diamond}$ : better than MR; $(\cdot)$ : better than Base; bold: better than Ep0; $*$ : better than Ep1M; $\dagger$ : better than EHC1; $\ddagger$ : better than EHC5. Exact solution $p$-values are based on pairwise chi-squared tests with Holm correction. Fitness and bloat $p$-values are based on pairwise Wilcoxon rank-sum tests.

\begin{tabular}{|c|c|c|c|c|c|}
\hline Problem & Method & $\begin{array}{r}\text { Exact Solutions } \\
(\%)\end{array}$ & $\begin{array}{r}\text { Median Best } \\
\text { Fitness }\end{array}$ & $\begin{array}{r}\text { Median Program } \\
\text { Size }\end{array}$ & $\begin{array}{r}\text { Median Convergence } \\
\text { Time (s) }\end{array}$ \\
\hline \multirow{7}{*}{$\begin{array}{l}\text { Bacterial } \\
\text { Respiration } 1\end{array}$} & MR & 0 & 0.21196 & $\mathrm{n} / \mathrm{a}$ & $\dagger \dagger^{*} 0.01$ \\
\hline & Base & 0 & ${ }^{\diamond} 0.14519$ & 15.61 & 38.77 \\
\hline & Ep0 & 0 & $\diamond 0.16068$ & $\dagger \ddagger 10.33$ & 35.35 \\
\hline & Ep1M & 0 & ${ }^{\diamond} 0.15657$ & $\dagger \ddagger 10.42$ & 50.42 \\
\hline & EHC1 & 0 & ${ }^{* \diamond} 0.14160$ & $\ddagger 11.91$ & 46.14 \\
\hline & EHC5 & 0 & ${ }^{* \diamond} 0.13907$ & 12.52 & 29.03 \\
\hline & EHC10 & 0 & $\dagger^{* \diamond} 0.13245$ & 13.00 & 52.35 \\
\hline \multirow{7}{*}{$\begin{array}{l}\text { Bacterial } \\
\text { Respiration } 2\end{array}$} & MR & 0 & 0.21273 & $\mathrm{n} / \mathrm{a}$ & $\dagger \dagger^{*} 0.01$ \\
\hline & Base & 0 & ${ }^{\diamond} 0.00785$ & 15.54 & 74.09 \\
\hline & Ep0 & 0 & ${ }^{\diamond} 0.00794$ & $\dagger \ddagger 10.29$ & 31.31 \\
\hline & Ep1M & 0 & $\diamond 0.00957$ & $\dagger \ddagger 10.29$ & 71.70 \\
\hline & EHC1 & 0 & ${ }^{* \diamond} 0.00741$ & $\ddagger 11.58$ & 94.61 \\
\hline & EHC5 & 0 & $\dagger^{* \diamond} 0.00634$ & 12.19 & 77.55 \\
\hline & EHC10 & 0 & $\dagger^{* \diamond} 0.00617$ & 12.19 & 75.22 \\
\hline \multirow{7}{*}{ Bar Magnet 1} & MR & 0 & 0.04057 & $\mathrm{n} / \mathrm{a}$ & $\dagger \dagger^{*} 0.01$ \\
\hline & Base & 2 & $\dagger^{* \diamond} 0.02351$ & 14.84 & 87.13 \\
\hline & Ep0 & 1 & $\diamond^{\diamond} 0.02866$ & $\dagger \ddagger 10.32$ & 74.07 \\
\hline & Ep1M & 2 & $\diamond^{\diamond} 0.03155$ & $\dagger \ddagger 10.35$ & 83.61 \\
\hline & EHC1 & 7 & ${ }^{* \diamond} 0.02733$ & $\ddagger 11.31$ & 48.95 \\
\hline & EHC5 & 7 & $\dagger^{* \diamond} 0.02242$ & 12.24 & *39.49 \\
\hline & $\mathrm{EHC} 10$ & 7 & $\dagger^{* \diamond} 0.02103$ & 12.64 & $* 50.79$ \\
\hline \multirow{7}{*}{ Bar Magnet 2} & $\mathrm{MR}$ & 0 & 1.19817 & $\mathrm{n} / \mathrm{a}$ & $\ddagger 0.01$ \\
\hline & Base & $\diamond_{18}$ & ${ }^{* \diamond} 0.01254$ & 15.51 & 68.79 \\
\hline & Ep0 & $\diamond_{16}$ & ${ }^{\diamond} 0.01502$ & $\dagger \ddagger 10.77$ & 61.54 \\
\hline & Ep1M & $\diamond 16$ & ${ }^{\diamond} 0.01729$ & $\dagger \ddagger 10.72$ & 81.35 \\
\hline & EHC1 & $*^{\diamond} 41$ & ${ }^{* \diamond} 0.00005$ & 11.59 & 68.86 \\
\hline & EHC5 & ${ }^{* \diamond} 45$ & ${ }^{* \diamond} 0.00000$ & 12.00 & 93.17 \\
\hline & EHC10 & ${ }^{* \diamond} 38$ & ${ }^{* \diamond} 0.00001$ & 12.34 & $\ddagger 80.07$ \\
\hline \multirow{7}{*}{ Glider 1} & $\mathrm{MR}$ & 0 & 4.52904 & $\mathrm{n} / \mathrm{a}$ & 0.01 \\
\hline & Base & $\diamond^{\diamond} 75$ & ${ }^{\diamond} 0.00000$ & 7.22 & 82.65 \\
\hline & Ep0 & ${ }^{\diamond} 77$ & ${ }^{\diamond} 0.00000$ & 5.07 & 91.69 \\
\hline & Ep1M & $\diamond 76$ & $\diamond_{0.00000}$ & 4.25 & 64.20 \\
\hline & EHC1 & ${ }^{* \diamond} 99$ & ${ }^{* \diamond} 0.00000$ & * 3.03 & 53.92 \\
\hline & EHC5 & $*^{\diamond} 100$ & ${ }^{* \diamond} 0.00000$ & * 3.26 & 73.33 \\
\hline & EHC10 & ${ }^{* \diamond} 100$ & ${ }^{* \diamond} 0.00000$ & 3.53 & 81.55 \\
\hline \multirow{7}{*}{ Glider 2} & $\mathrm{MR}$ & 0 & 0.53583 & $\mathrm{n} / \mathrm{a}$ & 0.01 \\
\hline & Base & 100 & ${ }^{\diamond} 0.00000$ & 2.76 & 19.89 \\
\hline & Ep0 & 100 & ${ }^{\diamond} 0.00000$ & $\ddagger 1.71$ & 7.61 \\
\hline & Ep1M & 100 & ${ }^{\diamond} 0.00000$ & $\ddagger 1.62$ & 6.11 \\
\hline & EHC1 & 100 & ${ }^{\diamond} 0.00000$ & $\ddagger 1.49$ & 4.99 \\
\hline & EHC5 & 100 & ${ }^{\diamond} 0.00000$ & 2.46 & 6.37 \\
\hline & EHC10 & 100 & ${ }^{\diamond} 0.00000$ & $\ddagger 1.57$ & 4.14 \\
\hline \multirow{7}{*}{$\begin{array}{l}\text { Lotka-Volterra } \\
1\end{array}$} & MR & 0 & 0.94071 & $\mathrm{n} / \mathrm{a}$ & $\dagger \dagger 0.01$ \\
\hline & Base & 7 & ${ }^{* \diamond} 0.04218$ & 16.30 & 48.83 \\
\hline & Ep0 & $\diamond 10$ & ${ }^{\diamond} 0.06897$ & $\dagger \ddagger 11.07$ & 83.16 \\
\hline & Ep1M & 8 & ${ }^{\diamond} 0.06805$ & $\dagger \ddagger 11.24$ & 59.40 \\
\hline & EHC1 & $\diamond 13$ & ${ }^{* \diamond} 0.00050$ & $\ddagger 12.71$ & 82.81 \\
\hline & EHC5 & ${ }^{* \diamond} 30$ & $\dagger^{* \diamond} 0.00003$ & 13.20 & 73.86 \\
\hline & EHC10 & $\dagger^{* \diamond} 43$ & $\dagger^{* \bullet} 0.00000$ & 13.18 & 67.98 \\
\hline
\end{tabular}


Table 5: Comparison of best-of-run results for the ODE benchmark problems. Statistical significance $(p<0.05)$ is denoted as follows: ${ }^{\diamond}$ : better than MR; $(\cdot)$ : better than Base; bold: better than Ep0; $*$ : better than Ep1M; $\dagger$ : better than EHC1; $\ddagger$ : better than EHC5. Exact solution $p$-values are based on pairwise chi-squared tests with Holm correction. Fitness and bloat $p$-values are based on pairwise Wilcoxon rank-sum tests.

\begin{tabular}{|c|c|c|c|c|c|}
\hline Problem & Method & $\begin{array}{r}\text { Exact Solutions } \\
(\%)\end{array}$ & $\begin{array}{r}\text { Median Best } \\
\text { Fitness }\end{array}$ & $\begin{array}{r}\text { Median Program } \\
\text { Size }\end{array}$ & $\begin{array}{r}\text { Median Convergence } \\
\text { Time (s) }\end{array}$ \\
\hline \multirow{7}{*}{$\begin{array}{l}\text { Lotka-Volterra } \\
2\end{array}$} & MR & 0 & 0.19556 & $\mathrm{n} / \mathrm{a}$ & 0.01 \\
\hline & Base & $\diamond_{53}$ & $\diamond 0.00000$ & 14.51 & 89.27 \\
\hline & Ep0 & $\diamond 73$ & ${ }^{\diamond} 0.00000$ & 6.33 & 59.00 \\
\hline & Ep1M & $\diamond 67$ & ${ }^{\diamond} 0.00000$ & 7.04 & 77.29 \\
\hline & $\mathrm{EHC} 1$ & ${ }^{* \diamond} 90$ & ${ }^{* \diamond} 0.00000$ & $* 4.77$ & 84.41 \\
\hline & EHC5 & ${ }^{* \diamond} 97$ & ${ }^{* \diamond} 0.00000$ & * 4.31 & 68.87 \\
\hline & EHC10 & ${ }^{* \diamond} 99$ & ${ }^{* \diamond} 0.00000$ & $\dagger^{*} \mathbf{3 . 1 9}$ & 76.08 \\
\hline \multirow{7}{*}{$\begin{array}{l}\text { Predator Prey } \\
1\end{array}$} & $\mathrm{MR}$ & 0 & 2.42447 & $\mathrm{n} / \mathrm{a}$ & $\dagger \dagger * 0.01$ \\
\hline & Base & 0 & ${ }^{* \diamond} 0.17383$ & 16.28 & 76.36 \\
\hline & Ep0 & 0 & ${ }^{\diamond} 0.19041$ & $\dagger \ddagger 10.89$ & 66.30 \\
\hline & Ep1M & 0 & ${ }^{\diamond} 0.19159$ & $\dagger \ddagger 10.89$ & 84.51 \\
\hline & EHC1 & 0 & ${ }^{* \diamond} 0.18286$ & $\ddagger 12.61$ & 62.70 \\
\hline & EHC5 & 0 & $\dagger^{* \diamond} 0.16950$ & 13.37 & 71.86 \\
\hline & EHC10 & 0 & $\dagger^{* \diamond} 0.16023$ & 13.54 & 79.33 \\
\hline \multirow{7}{*}{$\begin{array}{l}\text { Predator Prey } \\
2\end{array}$} & MR & 0 & 0.31338 & $\mathrm{n} / \mathrm{a}$ & $\dagger \dagger * 0.01$ \\
\hline & Base & 0 & $\dagger^{* \diamond} 0.20065$ & 16.72 & 86.67 \\
\hline & Ep0 & 0 & $\diamond 0.23531$ & $\dagger \ddagger 11.41$ & 90.11 \\
\hline & Ep1M & 0 & $\diamond^{\diamond} 0.23249$ & $\dagger \ddagger 11.38$ & $\dagger \ddagger 29.60$ \\
\hline & EHC1 & 0 & ${ }^{* \diamond} 0.20694$ & $\ddagger 12.86$ & 84.19 \\
\hline & EHC5 & 1 & $\dagger^{* \diamond} 0.19156$ & 13.69 & 85.89 \\
\hline & EHC10 & 0 & $\dagger^{* \diamond} 0.19136$ & 13.78 & 52.34 \\
\hline \multirow{7}{*}{ Shear Flow 1} & $\mathrm{MR}$ & 0 & 3.33019 & $\mathrm{n} / \mathrm{a}$ & 0.01 \\
\hline & Base & $\diamond 100$ & ${ }^{\diamond} 0.00000$ & $\dagger \ddagger 1.42$ & 9.50 \\
\hline & Ep0 & $\diamond 100$ & $\diamond^{\diamond} 0.00000$ & $\dagger \ddagger 1.35$ & 4.00 \\
\hline & Ep1M & $\diamond 100$ & ${ }^{\diamond} 0.00000$ & $\dagger \ddagger 1.16$ & 4.43 \\
\hline & EHC1 & $\diamond 100$ & $\diamond^{\diamond} 0.00000$ & $\ddagger 1.83$ & 2.70 \\
\hline & EHC5 & $\diamond^{\diamond} 100$ & ${ }^{\diamond} 0.00000$ & 2.17 & 2.00 \\
\hline & EHC10 & $\diamond 100$ & ${ }^{\diamond} 0.00000$ & $\dagger \ddagger 1.11$ & 3.43 \\
\hline \multirow{7}{*}{ Shear Flow 2} & MR & 0 & 0.56694 & $\mathrm{n} / \mathrm{a}$ & $\dagger \dagger^{*} 0.01$ \\
\hline & Base & 0 & ${ }^{\diamond} 0.00100$ & 16.68 & *32.31 \\
\hline & Ep0 & 1 & ${ }^{\diamond} 0.00111$ & $\dagger \ddagger 10.94$ & 104.38 \\
\hline & Ep1M & 0 & ${ }^{\diamond} 0.00099$ & $\dagger \ddagger 11.00$ & 106.03 \\
\hline & $\mathrm{EHC1}$ & 0 & ${ }^{* \diamond} 0.00092$ & $\ddagger 11.96$ & 76.78 \\
\hline & EHC5 & 1 & ${ }^{* \diamond} 0.00093$ & 12.32 & 83.49 \\
\hline & EHC10 & 0 & ${ }^{* \diamond} 0.00040$ & 12.41 & *90.77 \\
\hline \multirow{7}{*}{ van der Pol 1} & $\mathrm{MR}$ & 0 & 8.90324 & $\mathrm{n} / \mathrm{a}$ & $\dagger \dagger * 0.01$ \\
\hline & Base & 0 & ${ }^{* \diamond} 0.23003$ & 18.81 & 87.86 \\
\hline & Ep0 & 0 & ${ }^{\diamond} 0.24255$ & $\dagger \ddagger 13.87$ & 82.28 \\
\hline & Ep1M & 0 & ${ }^{\diamond} 0.27929$ & $\dagger \ddagger 13.95$ & 78.77 \\
\hline & EHC1 & 1 & ${ }^{* \diamond} 0.20830$ & 14.84 & 85.48 \\
\hline & EHC5 & 3 & $\dagger^{* \diamond} 0.10080$ & 14.95 & 69.16 \\
\hline & EHC10 & 0 & $\dagger^{* \diamond} 0.10159$ & 15.19 & 66.99 \\
\hline \multirow{7}{*}{ van der Pol 2} & MR & 100 & 0.00000 & $\mathrm{n} / \mathrm{a}$ & 0.01 \\
\hline & Base & 100 & 0.00000 & $\dagger^{*} 1.31$ & 4.21 \\
\hline & Ep0 & 100 & 0.00000 & 2.02 & 1.66 \\
\hline & Ep1M & 100 & 0.00000 & 1.51 & 2.35 \\
\hline & EHC1 & 100 & 0.00000 & 1.54 & 2.28 \\
\hline & EHC5 & 100 & 0.00000 & $\dagger^{*} \mathbf{1 . 3 4}$ & 3.34 \\
\hline & EHC10 & 100 & 0.00000 & 2.30 & 4.78 \\
\hline
\end{tabular}




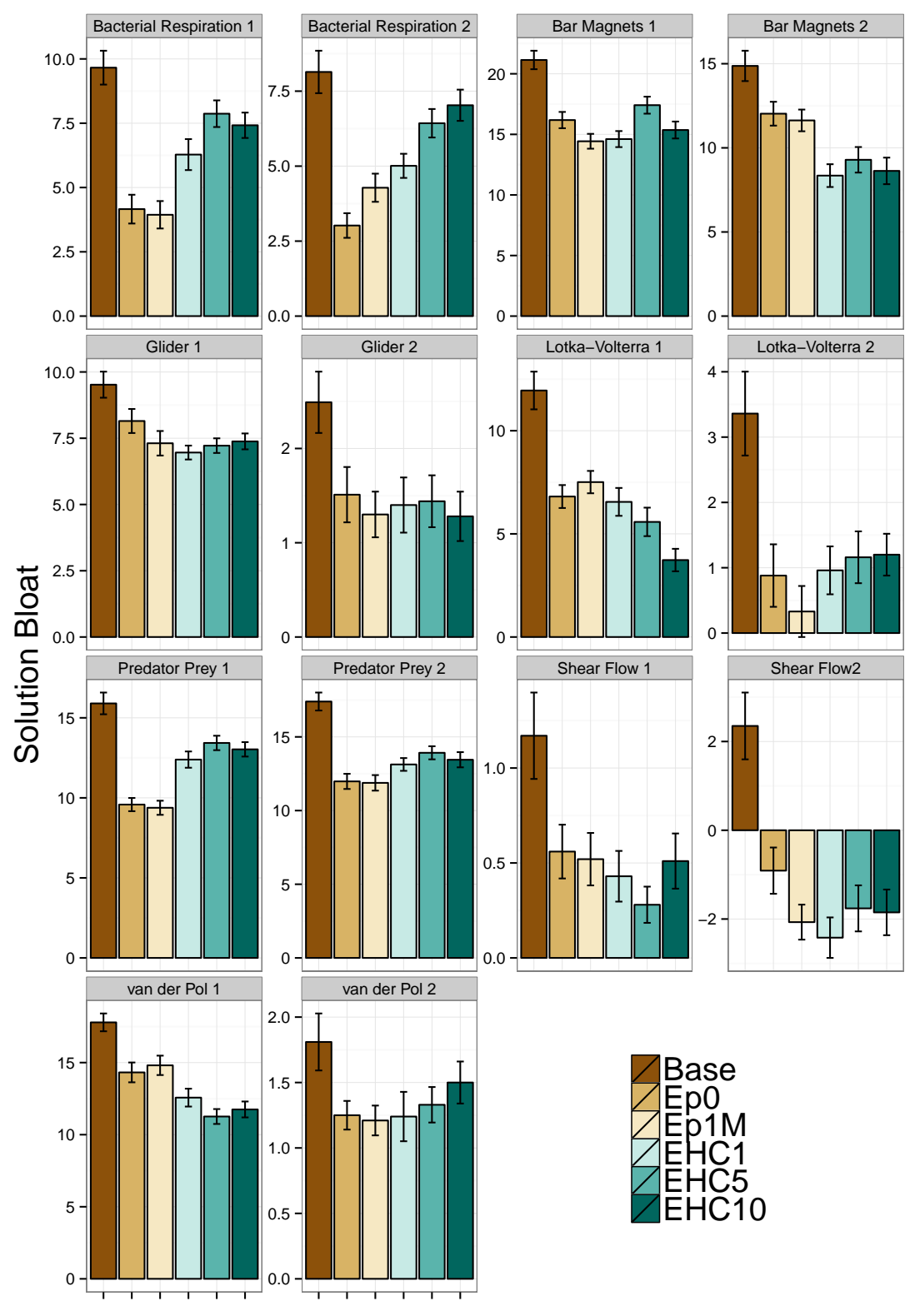

Figure 4: Comparison of solution bloat for the textbook ODE problems. 


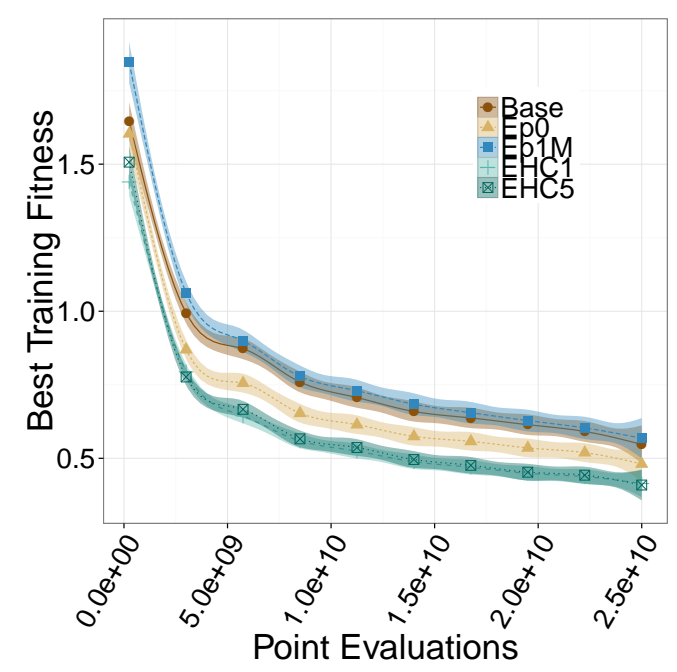

Figure 5: Fitness on the training set for the ODE suite.

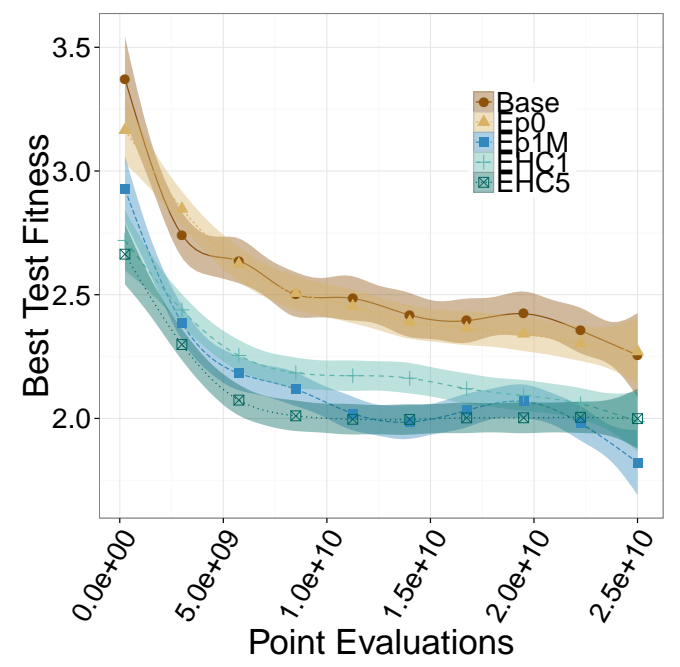

Figure 6: Fitness on the test set for the ODE suite.

The results also suggest that the computational effort improvement afforded by EHC increases with the complexity of the problem.

\subsection{Real-world Benchmark System}

In this section, EHC5 and several standard modeling approaches are applied to the identification of a benchmark system of cascaded tanks fed by a pump [65]. Since the true parameters of the system are not provided in [65], we first estimate the parameters of the model in Eq. (13) by linear regression, which yields

$$
\begin{aligned}
& \hat{\dot{y}}_{1}=-0.0122 \sqrt{\hat{y}_{1}}+0.0188 u(t) \\
& \hat{\dot{y}}_{2}=-0.0481 \sqrt{\hat{y}_{1}}+0.0452 \sqrt{\hat{y}_{2}}
\end{aligned}
$$

In comparison, EHC5 applied to the measured data yields

$$
\begin{array}{ll}
\hat{\dot{y}}_{1}= & -\hat{\theta}_{1} \sqrt{\hat{y}_{1}}+\hat{\theta}_{1} \sqrt{\hat{y}_{2}} \\
\hat{\dot{y}}_{2}= & -\hat{\theta}_{2} \sqrt{\hat{y}_{1}}+\hat{\theta}_{2}\left(u(t)+\hat{\theta}_{3}\right)
\end{array}
$$

where $\hat{\theta}_{1}=0.0905, \hat{\theta}_{2}=0.0302$, and $\hat{\theta}_{3}=0.6845$. The model in Eq. (15) is an interesting permutation of the theoretical model (Eq. (14)) in that it correctly identifies the square root nonlinearities of the water levels and is as succinct as the theoretical model. However Eq. (15) incorrectly associates the pump input $u(t)$ and top tank level $y_{1}(t)$ with the second state derivative, and uses the theoretical form of $\hat{\dot{y}}_{2}(t)$ in Eq. (14) for the first state derivative $\hat{\dot{y}}_{1}$ in Eq. (15). In comparison to the theoretical model, however, the ELGP solution produces much better predictions, as illustrated by the time series comparison in Figure 9 .

For this system, we compare the test set accuracy of the EHC5 model to some state-of-the-art black-box models in Table 6, in terms of the number of parameters in the resultant model, and the mean square error (MSE) and $R^{2}$ (Eq. (5)) of simulated outputs on the test set. The most 


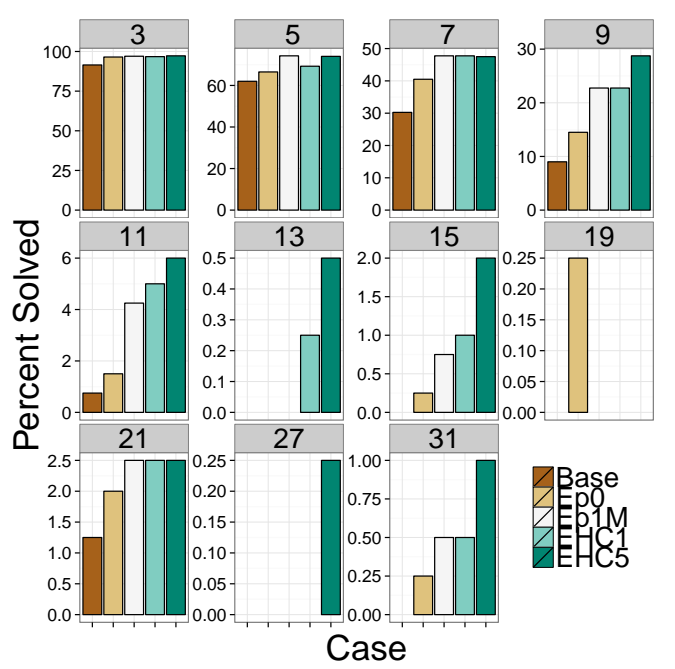

Figure 7: Percent of solutions found for the ODE suite. Results are grouped based on the number of nodes in the target equation (labelled at the top).

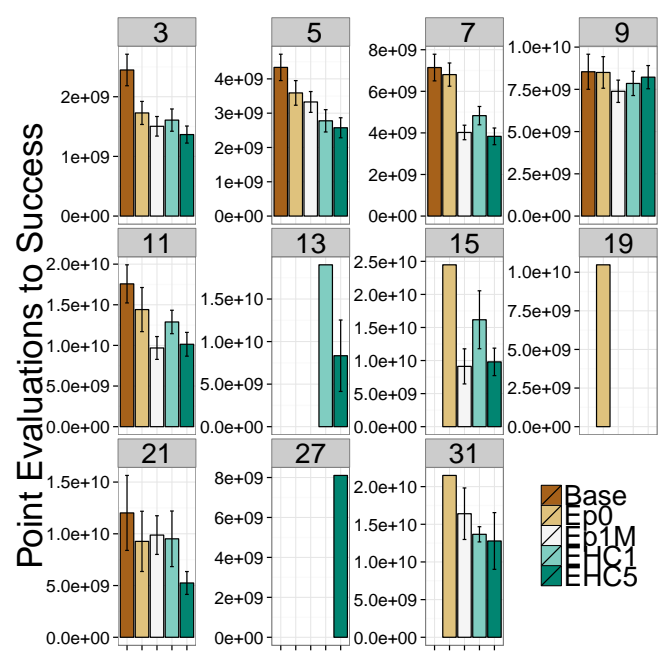

Figure 8: Point evaluations to success for the ODE suite. Results are grouped based on the number of nodes in the target equation (labelled at the top).

accurate model predictions are generated by NARX-NN, both in terms of MSE and $R^{2}$, followed by ELGP, which ties NARX-NN in prediction correlation for $y_{2}(t)$. ARX is the next most accurate on average, followed by the theoretical model and NARX-W. In comparison to the predictions of $\hat{y}_{1}$ and $\hat{y}_{2}$ from the ARX model, the ELGP model has a $42 \%$ and $18 \%$ lower mean absolute error, and $4 \%$ and $1 \%$ better $\mathrm{R}^{2}$ value. The inaccuracy of NARX-W is surprising given its complexity (64 parameters), and suggests a mismatch between the assumed nonlinearities of the approach and those present in the measured system. The NARX-NN model's excellent predictions come at the expense of complexity: the model consists of two networks with 10 hidden neurons each, totaling 60 learned parameters per model. In this sense the ELGP solution is quite reassuring because it achieves reasonable accuracy in prediction with half the parameters of the ARX model and 57 less parameters than the NARX-NN model.

The difficulty in estimating the correct model form for this system (Eq. (14)) may stem from the similarity of the measured outputs $y_{1}(t), y_{2}(t)$, as is shown in Figure 9 , as well as the effects of system noise and measurement error $\left(e_{1}(t), e_{1}(t), w_{1}(t), w_{2}(t)\right)$. It is especially clear from Figure 9 that the measured $y_{2}(t)$ deviates substantially from its theoretical behavior, making the theoretical model difficult to infer. More fundamentally, the identification difficulty could stem from an intrinsic difficulty of ELGP in handling processes of this form subject to the measured input conditions. To determine whether or not the intrinsic difficulty of this system affects ELGP's results, we simulate the theoretical system given in Eq. (14) using the measured input and use the resulting outputs $\left(\hat{y}_{1}(t), \hat{y}_{2}(t), u(t)\right)$ to train models using EHC5. In this case, EHC5 renders a nearly perfect model of the theoretical system:

$$
\begin{aligned}
& \hat{\dot{y}}_{1}=-\hat{\theta}_{1} \sqrt{\left(\hat{\theta}_{2} \hat{y}_{1}\right)}+\hat{\theta}_{1} u(t) \\
& \hat{\dot{y}}_{2}=-\hat{\theta}_{3} \sqrt{\hat{y}_{1}}+\hat{\theta}_{3} \sqrt{\left(\hat{y}_{2} / \hat{\theta}_{4}\right)}
\end{aligned}
$$



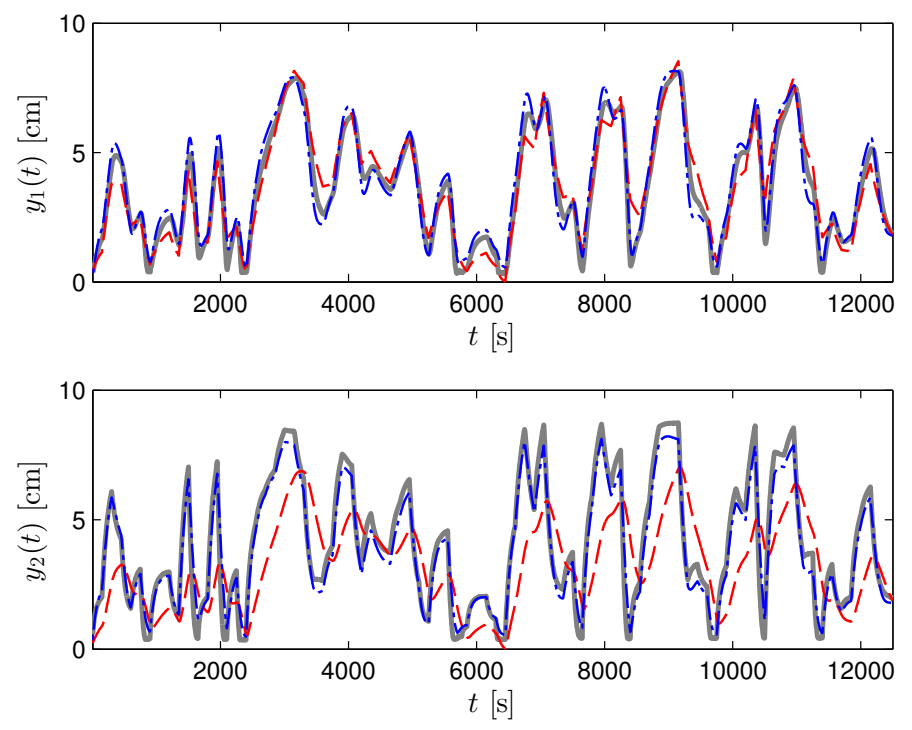

Figure 9: Comparison of outputs for the cascaded tanks problem, including measurement data (gray), the theoretical model (Eq. (14), red), and the ELGP model (Eq. (15), blue).

Table 6: Mean square error (MSE) and $R^{2}$ values on the test sets for the cascaded tanks problem using several modeling approaches.

\begin{tabular}{lrcccc}
\hline \multirow{2}{*}{ Method } & Parameters & \multicolumn{2}{c}{ MSE (test) } & \multicolumn{2}{c}{$R^{2}$ (test) } \\
& & $y_{1}(t)$ & $y_{2}(t)$ & $y_{1}(t)$ & $y_{2}(t)$ \\
\hline Theoretical & 3 & 0.463 & 5.343 & 0.907 & 0.313 \\
ARX & 6 & 0.432 & 0.350 & 0.919 & 0.961 \\
ELGP (EHC5) & 3 & 0.249 & 0.288 & 0.953 & 0.974 \\
NLARX-W & 64 & 2.960 & 6.165 & 0.807 & 0.748 \\
NLARX-NN & 60 & 0.120 & 0.182 & 0.977 & 0.974 \\
\hline
\end{tabular}

with $\hat{\theta}_{1}=0.0188, \hat{\theta}_{2}=0.4200, \hat{\theta}_{3}=0.0452$, and $\hat{\theta}_{4}=0.8830$. Both states in Eq. (16) have an MSE $<10^{-6}$ and $R^{2}=1$. Thus the mismatch between the ELGP model form and the assumed process physics appears to arise from system noise and measurement error.

\subsection{Industrial Processes}

The GP variants were used to identify models for two industrial processes: the Tower problem and Wind problem. We compare the fitness of the different GP methods as a function of computational effort (point evaluations) for the Tower and Wind problems in Fig. 10. For both problems, EHC1 converges most quickly to the minimum fitness found for the problems. For the Tower problem, we observe that Ep1M performs well on the test set, and as suggested in previous work [32], this may be due to the compact models produced by this method for this problem, since smaller models tend to generalize better than larger models. On the Wind problem, EHC1 and EHC5 converge the quickest and produce the best fitness on the test set. EpO and EHC10 tend to perform worse than EHC1, suggesting that EHC10 is overly greedy for these problems. Overall, the ELGP 


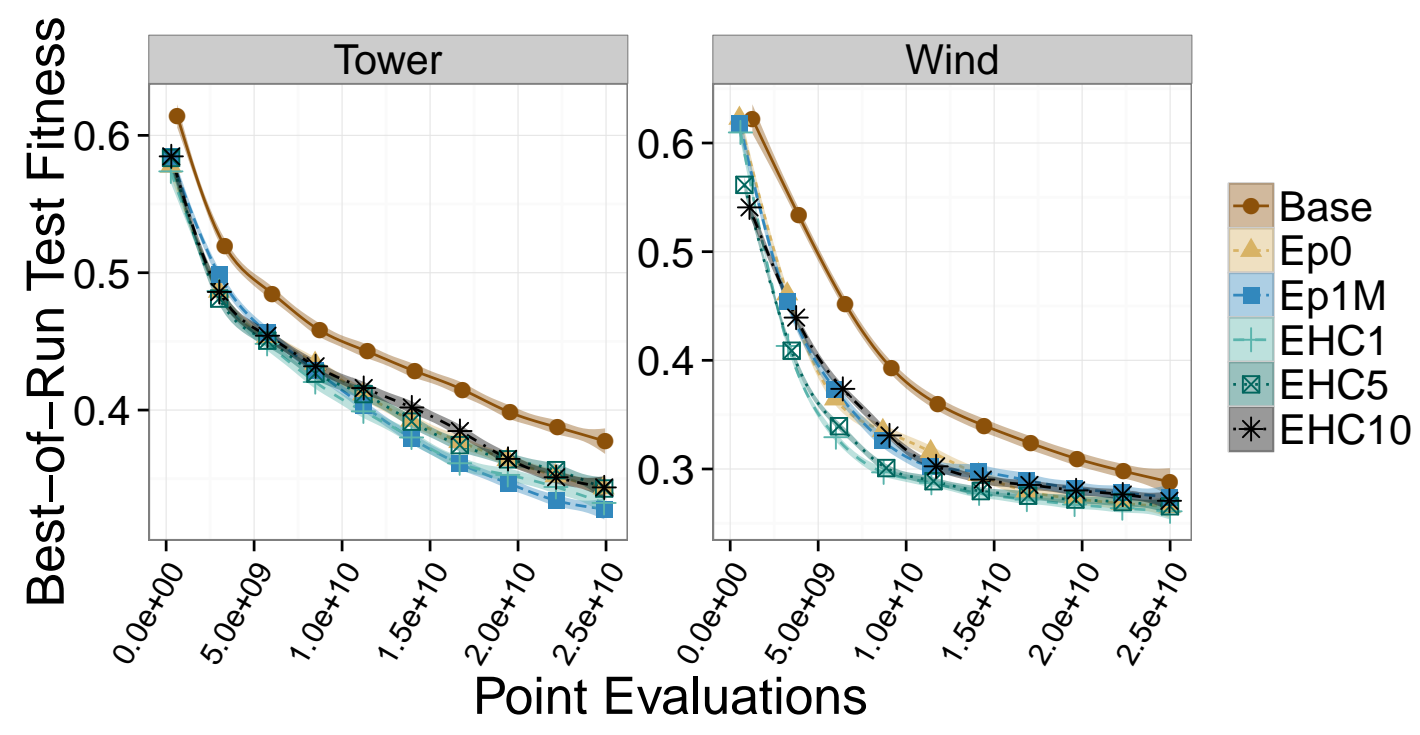

Figure 10: Fitness on the test set for the industrial problems. Filled area indicates the $95 \%$ confidence interval.

Table 7: Mean square error (MSE) and $R^{2}$ values of model predictions on the test sets for the Tower and Wind problem using several approaches.

\begin{tabular}{llrrr}
\hline Problem & Method & Parameters & MAE (test) & $\mathrm{R}^{2}$ (test) \\
\hline \multirow{3}{*}{ Tower } & ARX & 130 & 55.190 & 0.028 \\
& ELGP & 7 & 30.753 & 0.811 \\
& NARX-NN & 250 & 22.439 & 0.846 \\
\hline \multirow{3}{*}{ Wind } & ARX & 35 & 0.026 & 0.642 \\
& ELGP & 5 & 0.025 & 0.773 \\
& NARX-NN & 60 & 0.026 & 0.727 \\
\hline
\end{tabular}

variants perform significantly better than Base on both problems, converging approximately twice as fast as Base and producing better fit models.

We compare the best ELGP models to models identified using ARX and NARX-NN in Table 7. For the Tower problem, the ELGP model is a notable improvement over the linear model (note the $279 \%$ higher $\mathrm{R}^{2}$ value), but is slightly less accurate than the model generated using NARX-NN ( $4 \%$ lower $\mathrm{R}^{2}$ ). However it is important to note that the high number of variables in this problem produce a neural network with 250 parameters, whereas the ELGP model contains only 7 . For the Wind problem, ELGP produces the most accurate model among the tested methods. The MAE of the models generated by each method are similar; however, the correlation of the ELGP model to test data is $20 \%$ better than the ARX model, and $6 \%$ better than the NARX-NN model. The ELGP model also contains significantly fewer parameters than the ARX or NARX-NN models. Hence the conciseness of the ELGP models is an encouraging trade-off for the accuracy they exhibit. 


\subsection{Population Diversity}

Results on the textbook ODEs and the ODE suite suggest that the EHC methods improve GP performance significantly. We hypothesize that this improvement is created by preserving sections of the genome from fitness pressure and allowing them to drift genetically, thus providing an avenue for introduction of diversity and continued progress towards the solution. To test this hypothesis, the syntactic and semantic similarity of models in the population can be examined in detail to determine whether this phenomenon of preserved diversity is evident. We define syntactic similarity as the homology $H$ of a population using a Levenshtein distance comparison of $S$ randomly sampled pairs of individuals $\left(\left|\mathbf{i}_{j}, \mathbf{i}_{m}\right|_{L}\right)$ normalized by the length $(|\cdot|)$ of the longer individual, as

$$
H=1-\frac{1}{S} \sum_{n=1}^{S} \frac{\left|\mathbf{i}_{j}, \mathbf{i}_{m}\right|_{L}}{\max \left(\left|\mathbf{i}_{j}\right|,\left|\mathbf{i}_{m}\right|\right)}
$$

We sample $H$ each generation for both the active and inactive portions of genomes with $S=200$. In addition, we define the semantic; i.e., behavioral, similarity of the population, referred to as Similar Behavior, as the fraction of identical output vectors among models in the population. This allows us to compare what is happening genetically and epigenetically at the program level (syntax) to the behavior of the resultant models (semantics).

In general we find that silenced genotypes have lower homology (i.e. higher diversity) than expressed genotypes for every epigenetic treatment, thus demonstrating that genetic drift is indeed occurring in inactive sections of programs. For instance, we measured $H$ for the Bacterial Respiration problem (state 2) for each treatment, the results of which are shown in Figure 11. Homology in the expressed genome is more or less equivalent for all treatments. Despite having similar expressed genetic homologies, we find that behavioral similarity increases more quickly with the hill climbing methods (EHC1, EHC5, EHC10) than with Base, Ep0 or Ep1M, as shown in Figure 12. To understand why the EHC methods converge the quickest semantically, recall that these methods only preserve epigenetic mutations that improve semantics, and are therefore more greedy than the other methods. Since the best methods for this problem converge on Similar Behavior the quickest, it appears that greedy topological search afforded by the EHC methods is an important factor in creating the improvements noted in our experiments. From the perspective of search, Figures 11 and 12 imply that the EHC systems are exploiting neutral variation in the genome as well as improved reachability in the genotype-phenotype mapping provided by epigenetics, since $H$ remains flat while Similar Behavior increases. Neutral variation is a property known to benefit other GP methods as well [60]. In other words, the epigenetic systems converge on model behavior more quickly while preserving genetic diversity in the search space.

It is important to note that the smoothness of the fitness landscape of a problem will play a role in determining whether greedy methods like EHC are the best option. For example, we studied several program synthesis problems for which Ep1M provided better performance [32]. This could be due to rugged and/or deceptive fitness landscapes. Given that the EHC methods work best for the dynamic systems studied in this paper, it is likely that the fitness landscapes are less deceptive than those for program synthesis. It is also likely that similar systems in this domain have similar properties, and they should therefore benefit from the EHC variants of ELGP as well.

\section{Conclusions}

The results suggest that epigenetic local search is a significant addition to GP. We find that epigenetic methods, especially EHC methods, outperform a baseline implementation of GP in terms 


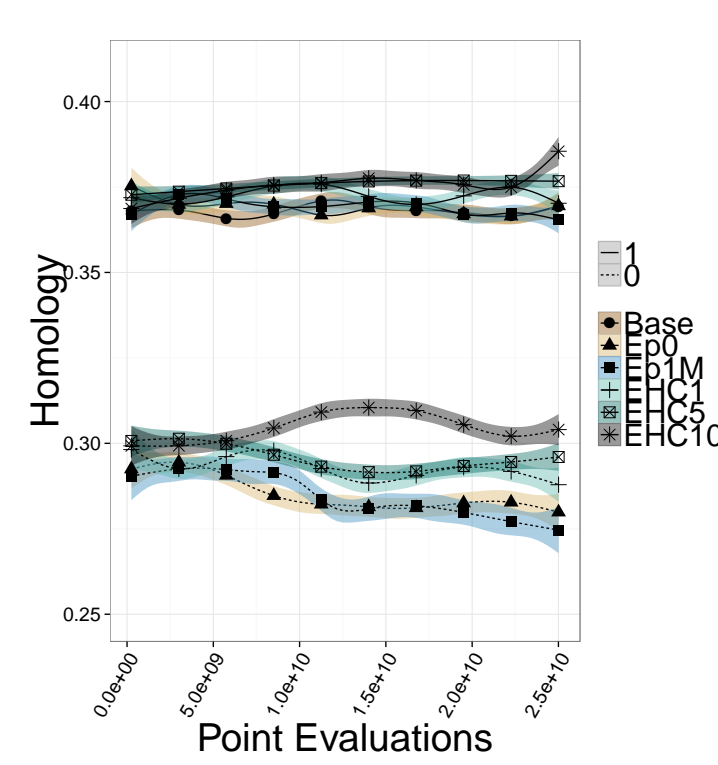

Figure 11: Homology among active and inactive genomes for the Bacterial Respiration 2 problem.

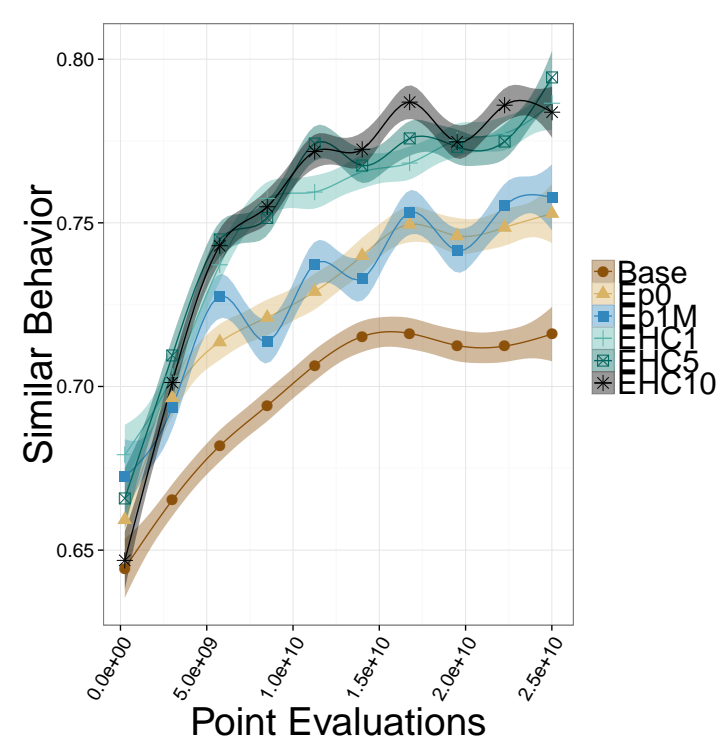

Figure 12: Similar Behavior (fraction of unique output vectors in the population) for the Bacterial Respiration 2 problem.

of fitness minimization, exact solutions, and equation intelligibility on textbook nonlinear ODE systems and randomly generated dynamic systems. Furthermore we show in comparison to other nonlinear approaches that ELGP is able to return concise and accurate models in three real-world applications. Our study of population diversity suggests that this performance improvement to GP is achieved by the epigenetic layer's ability to preserve diversity in the inactive sequences of genes while converging more quickly in semantic space. Although we have only considered epigenetic learning by mutation and hill climbing here, the results encourage further research into the use of epigenetic methods for structure optimization in GP, and motivate a focus on methods that improve the ability of GP to search equation topologies, in addition to constants.

\section{Acknowledgments}

We thank Nicholas McPhee and the Hampshire Computational Intelligence lab for helping improve this paper. This work is partially supported by the NSF-sponsored IGERT: Offshore Wind Energy Engineering, Environmental Science, and Policy (Grant Number 1068864), as well as Grant No. 1017817, 1129139, and 1331283. Any opinions, findings, and conclusions or recommendations expressed in this publication are those of the authors and do not necessarily reflect the views of the National Science Foundation. This work used the Extreme Science and Engineering Discovery Environment (XSEDE), which is supported by National Science Foundation grant number ACI$1053575[59]$.

[1] I. Arnaldo, K. Krawiec, and U.-M. O'Reilly. Multiple regression genetic programming. In Proceedings of the 2014 conference on Genetic and evolutionary computation, pages 879-886. ACM Press, 2014. 
[2] W. Banzhaf. Genotype-phenotype-mapping and neutral variation - a case study in genetic programming. In Parallel problem solving from nature (PPSN) III, pages 322-332. Springer, 1994.

[3] S. A. Billings. Nonlinear system identification: NARMAX methods in the time, frequency, and spatio-temporal domains. John Wiley \& Sons, 2013.

[4] J. Bongard and H. Lipson. Automated reverse engineering of nonlinear dynamical systems. Proceedings of the National Academy of Sciences, 104(24):9943-9948, 2007.

[5] M. Brameier and W. Banzhaf. Linear Genetic Programming, volume 1. Springer, 1 edition, 2007.

[6] H. Cao, L. Kang, Y. Chen, and J. Yu. Evolutionary modeling of systems of ordinary differential equations with genetic programming. Genetic Programming and Evolvable Machines, 1(4):309337,2000 .

[7] S. Chen, S. A. Billings, and W. Luo. Orthogonal least squares methods and their application to non-linear system identification. International Journal of control, 50(5):1873-1896, 1989.

[8] T. W. Cornforth and H. Lipson. Inference of hidden variables in systems of differential equations with genetic programming. Genet Program Evolvable Mach, 14(2):155-190, June 2013.

[9] K. Deb, S. Agrawal, A. Pratap, and T. Meyarivan. A Fast Elitist Non-dominated Sorting Genetic Algorithm for Multi-objective Optimization: NSGA-II. In M. Schoenauer, K. Deb, G. Rudolph, X. Yao, E. Lutton, J. J. Merelo, and H.-P. Schwefel, editors, Parallel Problem Solving from Nature PPSN VI, volume 1917, pages 849-858. Springer Berlin Heidelberg, Berlin, Heidelberg, 2000.

[10] B. G. Dias and K. J. Ressler. PACAP and the PAC1 Receptor in Post-Traumatic Stress Disorder. Neuropsychopharmacology, 38(1):245-246, Jan. 2013.

[11] B. G. Dias and K. J. Ressler. Parental olfactory experience influences behavior and neural structure in subsequent generations. Nat Neurosci, 17(1):89-96, Jan. 2014.

[12] C. Ferreira. Gene Expression Programming: a New Adaptive Algorithm for Solving Problems. arXiv:cs/0102027, Feb. 2001. Complex Systems, 13(2): 87-129, 2001.

[13] P. Fleming, J.-W. Van Wingerden, and A. D. Wright. Comparing State-space Multivariable Controls to Multi-SISO Controls for Load Reduction of Drivetrain-coupled Modes on Wind Turbines Through Field-testing: Preprint. National Renewable Energy Laboratory,[National Wind Technology Center, 2011.

[14] A. Fontana. Epigenetic Tracking: Biological Implications. In G. Kampis, I. Karsai, and E. Szathmry, editors, Advances in Artificial Life. Darwin Meets von Neumann, number 5777 in Lecture Notes in Computer Science, pages 10-17. Springer Berlin Heidelberg, Jan. 2011.

[15] C. Giraud-Carrier. Unifying Learning with Evolution Through Baldwinian Evolution and Lamarckism. In Advances in Computational Intelligence and Learning, pages 159-168. Springer, 2002. 
[16] G. J. Gray, D. J. Murray-Smith, Y. Li, K. C. Sharman, and T. Weinbrenner. Nonlinear model structure identification using genetic programming. Control Engineering Practice, 6(11):1341$1352,1998$.

[17] G. Gregori and G. Lightbody. Nonlinear system identification: From multiple-model networks to Gaussian processes. Engineering Applications of Artificial Intelligence, 21(7):1035-1055, Oct. 2008.

[18] F. Gruau and D. Whitley. Adding Learning to the Cellular Development of Neural Networks: Evolution and the Baldwin Effect. Evolutionary Computation, 1(3):213-233, Sept. 1993.

[19] R. Haber and H. Unbehauen. Structure identification of nonlinear dynamic systemsa survey on input/output approaches. Automatica, 26(4):651-677, 1990.

[20] R. Holliday. Epigenetics: a historical overview. Epigenetics, 1(2):76-80, 2006.

[21] H. Iba and T. Sato. Genetic Programming with Local Hill-Climbing. Technical Report ETLTR-94-4, Electrotechnical Laboratory, 1-1-4 Umezono, Tsukuba-city, Ibaraki, 305, Japan, 1994.

[22] E. Jablonka and M. J. Lamb. The changing concept of epigenetics. Annals of the New York Academy of Sciences, 981(1):82-96, 2002.

[23] I.-k. Jeong and J.-j. Lee. Adaptive simulated annealing genetic algorithm for system identification. Engineering Applications of Artificial Intelligence, 9(5):523-532, Oct. 1996.

[24] G. Kaati, L. O. Bygren, and S. Edvinsson. Cardiovascular and diabetes mortality determined by nutrition during parents' and grandparents' slow growth period. European Journal of Human Genetics, 10(11):682, Nov. 2002.

[25] M. Keijzer. Improving Symbolic Regression with Interval Arithmetic and Linear Scaling. In C. Ryan, T. Soule, M. Keijzer, E. Tsang, R. Poli, and E. Costa, editors, Genetic Programming, number 2610 in Lecture Notes in Computer Science, pages 70-82. Springer Berlin Heidelberg, Jan. 2003.

[26] M. Keijzer. Push-forth: A Light-weight, Strongly-typed, Stack-based Genetic Programming Language. In Proceedings of the 15th Annual Conference Companion on Genetic and Evolutionary Computation, GECCO '13 Companion, pages 1635-1640, New York, NY, USA, 2013. ACM.

[27] M. Kommenda, G. Kronberger, M. Affenzeller, S. M. Winkler, and B. Burlacu. Evolving Simple Symbolic Regression Models by Multi-objective Genetic Programming. In Genetic Programming Theory and Practice, volume XIV of Genetic and Evolutionary Computation. Springer, Ann Arbor, MI, 2015.

[28] M. Kommenda, G. Kronberger, S. Winkler, M. Affenzeller, and S. Wagner. Effects of constant optimization by nonlinear least squares minimization in symbolic regression. In C. Blum, E. Alba, T. Bartz-Beielstein, D. Loiacono, F. Luna, J. Mehnen, G. Ochoa, M. Preuss, E. Tantar, and L. Vanneschi, editors, GECCO '13 Companion: Proceeding of the fifteenth annual conference companion on Genetic and evolutionary computation conference companion, pages 1121-1128, Amsterdam, The Netherlands, 2013. ACM. 
[29] J. R. Koza. Genetic Programming: On the Programming of Computers by Means of Natural Selection. MIT Press, Cambridge, MA, USA, 1992.

[30] J. R. Koza, F. H. Bennett III, D. Andre, M. A. Keane, and F. Dunlap. Automated synthesis of analog electrical circuits by means of genetic programming. Evolutionary Computation, IEEE Transactions on, 1(2):109-128, 1997.

[31] W. La Cava, K. Danai, L. Spector, P. Fleming, A. Wright, and M. Lackner. Automatic identification of wind turbine models using evolutionary multiobjective optimization. Renewable Energy, Nov. 2015.

[32] W. La Cava, T. Helmuth, L. Spector, and K. Danai. Genetic Programming with Epigenetic Local Search. In Proceedings of the Genetic and Evolutionary Computation Conference (GECCO), pages 1055-1062. ACM Press, 2015.

[33] W. La Cava, L. Spector, K. Danai, and M. Lackner. Evolving differential equations with developmental linear genetic programming and epigenetic hill climbing. In Companion proceedings of the 2014 conference on Genetic and evolutionary computation (GECCO), pages 141-142. ACM Press, 2014.

[34] W. G. La Cava and K. Danai. Gradient-based adaptation of continuous dynamic model structures. International Journal of Systems Science, 0(0):1-15, Aug. 2015.

[35] W. B. Langdon. Genetic programming and data structures: genetic programming + data structures= automatic programming!, volume 1. Springer Science \& Business Media, 2012.

[36] L. Ljung. System Identification: Theory for the User, volume 1. Prentice Hall, 2nd ed. edition, 1999.

[37] L. Ljung. System Identification Toolbox for Use with $\$\{\$$ matlab $\$\} \$ 2007$.

[38] J. Madar, J. Abonyi, and F. Szeifert. Genetic programming for system identification. In Intelligent Systems Design and Applications (ISDA 2004) Conference, Budapest, Hungary, 2004.

[39] J. F. Miller and P. Thomson. Cartesian genetic programming. In Genetic Programming, pages 121-132. Springer, 2000.

[40] K. S. Narendra and K. Parthasarathy. Identification and control of dynamical systems using neural networks. Neural Networks, IEEE Transactions on, 1(1):4-27, 1990.

[41] X. Ni, M. Verhaegen, A. J. Krijgsman, and H. B. Verbruggen. A new method for identification and control of nonlinear dynamic systems. Engineering Applications of Artificial Intelligence, 9(3):231-243, June 1996.

[42] P. Nordin, F. Francone, and W. Banzhaf. Explicitly Defined Introns and Destructive Crossover in Genetic Programming. In J. P. Rosca, editor, Proceedings of the Workshop on Genetic Programming: From Theory to Real-World Applications, pages 6-22, Tahoe City, California, USA, 1995. 
[43] T. Perkis. Stack-based genetic programming. In Evolutionary Computation, 1994. IEEE World Congress on Computational Intelligence., Proceedings of the First IEEE Conference on, pages 148-153. IEEE, 1994.

[44] K. Rodrguez-Vzquez and P. J. Fleming. Evolution of mathematical models of chaotic systems based on multiobjective genetic programming. Knowledge and Information Systems, 8(2):235256, Oct. 2004.

[45] K. Rodriguez-Vazquez, C. M. Fonseca, and P. J. Fleming. 'Identifying the structure of nonlinear dynamic systems using multiobjective genetic programming. IEEE Transactions on Systems, Man, and Cybernetics - Part A: Systems and Humans, 34(4):531-545, July 2004.

[46] B. J. Ross. A Lamarckian evolution strategy for genetic algorithms. Practical handbook of genetic algorithms: complex coding systems, 3:1-16, 1999.

[47] A. Sadollah, H. Eskandar, D. G. Yoo, and J. H. Kim. Approximate solving of nonlinear ordinary differential equations using least square weight function and metaheuristic algorithms. Engineering Applications of Artificial Intelligence, 40:117-132, Apr. 2015.

[48] M. Schmidt and H. Lipson. Comparison of Tree and Graph Encodings As Function of Problem Complexity. In Proceedings of the 9th Annual Conference on Genetic and Evolutionary Computation, GECCO '07, pages 1674-1679, New York, NY, USA, 2007. ACM.

[49] M. Schmidt and H. Lipson. Distilling free-form natural laws from experimental data. Science, 324(5923):81-85, 2009.

[50] M. Schmidt and H. Lipson. Age-fitness pareto optimization. In Genetic Programming Theory and Practice VIII, pages 129-146. Springer, 2011.

[51] M. D. Schmidt. Machine Science: Automated Modeling of Deterministic and Stochastic Dynamical Systems. PhD thesis, Cornell University, Ithaca, NY, USA, 2011. AAI3484909.

[52] B. W. Silverman. Density estimation for statistics and data analysis, volume 26. CRC press, 1986.

[53] G. F. Smits and M. Kotanchek. Pareto-front exploitation in symbolic regression. In Genetic Programming Theory and Practice II, pages 283-299. Springer, 2005.

[54] L. Spector and T. Helmuth. Uniform linear transformation with repair and alternation in genetic programming. Genetic Programming Theory and Practice XI, page In preparation. Springer, 2013.

[55] L. Spector and A. Robinson. Genetic programming and autoconstructive evolution with the push programming language. Genetic Programming and Evolvable Machines, 3(1):7-40, 2002.

[56] S. H. Strogatz. Nonlinear dynamics and chaos: with applications to physics, biology, chemistry, and engineering. Westview press, 2014.

[57] I. Tanev and K. Yuta. Epigenetic programming: Genetic programming incorporating epigenetic learning through modification of histones. Information Sciences, 178(23):4469-4481, Dec. 2008. 
[58] A. Topchy and W. F. Punch. Faster genetic programming based on local gradient search of numeric leaf values. In Proceedings of the Genetic and Evolutionary Computation Conference (GECCO-2001), pages 155-162, 2001.

[59] J. Towns, T. Cockerill, M. Dahan, I. Foster, K. Gaither, A. Grimshaw, V. Hazlewood, S. Lathrop, D. Lifka, G. D. Peterson, R. Roskies, J. R. Scott, and N. Wilkens-Diehr. XSEDE: Accelerating Scientific Discovery. Computing in Science and Engineering, 16(5):62-74, 2014.

[60] A. J. Turner and J. F. Miller. Neutral genetic drift: an investigation using Cartesian Genetic Programming. Genet Program Evolvable Mach, pages 1-28, May 2015.

[61] B. M. Turner. Histone acetylation and an epigenetic code. Bioessays, 22(9):836-845, 2000.

[62] E. Vladislavleva, G. Smits, and D. den Hertog. Order of Nonlinearity as a Complexity Measure for Models Generated by Symbolic Regression via Pareto Genetic Programming. IEEE Transactions on Evolutionary Computation, 13(2):333-349, 2009.

[63] D. Whitley, V. S. Gordon, and K. Mathias. Lamarckian evolution, the Baldwin effect and function optimization. In Parallel Problem Solving from Nature (PPSN) III, pages 5-15. Springer, 1994.

[64] T. Wigren. Recursive prediction error identification and scaling of non-linear state space models using a restricted black box parameterization. Automatica, 42(1):159-168, Jan. 2006.

[65] T. Wigren. Input-output data sets for development and benchmarking in nonlinear identification. Technical Reports from the department of Information Technology, 20:2010020, 2010. Data sets available: http://www.it.uu.se/research/publications/reports/2010020/NonlinearData.zip.

[66] T. Wigren and J. Schoukens. Three free data sets for development and benchmarking in nonlinear system identification. In Proc. 2013 Eur. Control Conf.(ECC2013), pages 17-19, 2013.

[67] A. S. Wu and R. K. Lindsay. Empirical Studies of the Genetic Algorithm with Noncoding Segments. Evolutionary Computation, 3(2):121-147, June 1995.

[68] E. Zitzler, M. Laumanns, and L. Thiele. SPEA2: Improving the strength Pareto evolutionary algorithm. Eidgenössische Technische Hochschule Zürich (ETH), Institut für Technische Informatik und Kommunikationsnetze (TIK), 2001. 